Health Services Research

\title{
Declining Utilization and Inflation-Adjusted Expenditures for Epidural Procedures in Chronic Spinal Pain in the Medicare Population
}

\author{
Laxmaiah Manchikanti, MD', Vidyasagar Pampati, MSc', Amol Soin, MD², \\ Mahendra R. Sanapati, MD', Alan D. Kaye, MD, PhD³, and Joshua A. Hirsch, MD ${ }^{4}$
}

From: ${ }^{\text {PPain Management }}$ Centers of America, Paducah, $\mathrm{KY}$ and Evansville, IN; ${ }^{2} \mathrm{Ohio}$ Pain Clinic, Centerville, $\mathrm{OH}$,

Wright State University,

Dayton, OH; ${ }^{\mathrm{L}} \mathrm{SU}$ School

of Medicine, Shreveport,

LA and New Orleans, LA;

${ }^{4}$ Massachusetts General Hospital and Harvard Medical School, Boston, MA

Address Correspondence: Laxmaiah Manchikanti, MD Pain Management Centers of America

67 Lakeview Drive

Paducah, KY 42001

E-mail:drlm@thepainmd.com

Disclaimer: There was no external funding in the preparation of this manuscript.

Conflict of interest: Dr. Kaye is a speaker for Merck. Dr. Hirsch is a consultant for Medtronic and Senior Affiliate Research Fellow at the Neiman Policy Institute. Dr. Soin is the founder and CEO of Soin Neuroscience, which

is developing a spinal cord stimulator to treat spinal pain.

Manuscript received: 10/1/2020

Accepted for publication: $11 / 22 / 2020$

Free full manuscript: www.painphysicianjournal.com
Background: Despite epidurals being one of the most common interventional pain procedures for managing chronic spinal pain in the United States, expenditure analysis lacks assessment in correlation with utilization patterns.

Objectives: This investigation was undertaken to assess expenditures for epidural procedures in the feefor-service (FFS) Medicare population from 2009 to 2018.

Study Design: The present study was designed to assess expenditures in all settings, for all providers in the FFS Medicare population from 2009 to 2018 in the United States. In this manuscript:

- A patient was described as receiving epidural procedures throughout the year.

- A visit was considered to include all regions treated during the visit.

- An episode was considered as one treatment per region utilizing primary codes only.

- Services or procedures were considered as all procedures including bilateral and multiple levels.

A standard $5 \%$ national sample of the Centers for Medicare and Medicaid Services (CMS) physician outpatient billing claims data for those enrolled in the FFS Medicare program from 2009 to 2018 was utilized. All the expenditures were presented with allowed costs and adjusted to inflation to 2018 US dollars.

Results: Total expenditures were $\$ 723,981,594$ in 2009, whereas expenditures of 2018 were $\$ 829,987,636$, with an overall $14.6 \%$ increase, or an annual increase of $1.5 \%$. However, the inflationadjusted rate was $\$ 847,058,465$ in 2009 , compared to $\$ 829,987,636$ in 2018 , a reduction overall of $2 \%$ and an annual reduction of $0.2 \%$. Inflation-adjusted per patient annual costs decreased from $\$ 988.93$ in 2009 to $\$ 819.27$ in 2018 with a decrease of $17.2 \%$ or an annual decline of $2.1 \%$. In addition, inflationadjusted costs per procedure decreased from $\$ 399.77$ to $\$ 377.94$, or $5.5 \%$ overall and $0.6 \%$ annually.

Per procedure, episode, visit, and patient expenses were higher for transforaminal epidural procedures than lumbar interlaminar/caudal epidural procedures. Overall, costs of transforaminal epidurals increased $27.6 \%$ or $2.7 \%$ annually, whereas lumbar interlaminar and caudal epidural injections cost were reduced $2.7 \%$, or $0.3 \%$ annually. Inflation-adjusted costs for transforaminal epidurals increased $9.1 \%$ or $1.0 \%$ annually and declined 16.9 or $2.0 \%$ annually for lumbar interlaminar and caudal epidural injections.

Limitations: Expenditures for epidural procedures in chronic spinal pain were assessed only in the FFS Medicare population. This excluded over $30 \%$ of the Medicare population, which is enrolled in Medicare Advantage plans.

Conclusions: After adjusting for inflation, there was a decrease of expenditures for epidural procedures of $2 \%$, or $0.2 \%$ annually, from 2009 to 2018 . However, prior to inflation, the increases were noted at $14.6 \%$ and $1.5 \%$. Inflation-adjusted costs per patient, per visit, and per procedure also declined. The proportion of Medicare patients per 100,000 receiving epidural procedures decreased $9.1 \%$, or $1.1 \%$ annually. However, assessment of individual procedures showed higher costs for transforaminal epidural procedures compared to lumbar interlaminar and caudal epidural procedures.

Key words: Chronic spinal pain, epidural procedures, caudal epidural, lumbar interlaminar epidural, cervical interlaminar epidural, thoracic interlaminar epidural, lumbar transforaminal epidural procedures, Medicare expenditures

Pain Physician 2021: 24:1-15 
nterventional techniques have been a focus of utilization trends and subsequent increases in costs in recent years, accompanied by the application of multiple regulations and measures to reduce utilization and expenditures (1-8). One of the measures utilized for these purposes has been the enactment of the Affordable Care Act (ACA) to improve access, quality of care, and to reduce health care costs in the United States (9-11). However, the real effectiveness of these measures to control costs and the overall effectiveness of the ACA, which was signed into law in March of 2010, has been questioned in its ability to fulfill these objectives (10).

In fact, Peterson (12), in a manuscript of "The ACA a Decade in: Resilience, Impact, and Vulnerabilities," described that possession of an insurance card does not mean that one is well insured and without barriers to needed health care services. Further, he also concluded that the ACA had done nothing to reduce the aggregate percentage of what the Commonwealth Fund identifies as the "underinsured" - those facing out of pocket costs so significant that they lead to foregoing primary or specialist medical care, missing treatments, or not filling prescriptions. With extensive financial vulnerabilities, as many as $30 \%$ of the elderly forego care due to the expenses. In fact, US health care spending continues to increase and reached $\$ 3.8146$ trillion in 2019 (13). In addition, the national health expenditure survey estimated an average annual growth rate of $5.4 \%$ from 2019 to 2028.

Manchikanti et al (14) published an analysis of utilization trends and Medicare expenditures of spinal interventional techniques from 2000 to 2008. The data showed that Medicare recipients receiving spinal interventional techniques increased $186.8 \%$, at an annual rate increase of $14.1 \%$ per 100,000 fee-forservice (FFS) Medicare beneficiaries. Overall, approved amounts throughout the FFS population in the US were $\$ 362,347,025$ in 2000 compared to $\$ 1,231,180,420$ in 2008 , a $240 \%$ increase for all spinal interventional techniques.

In addition to these data, estimates continue to be onerous $(15,16)$. A recent follow up study on US spending on personal health and public health care from 1996 to 2016, showed an estimated spending of $\$ 129.8$ billion on other musculoskeletal disorders with $\$ 134.5$ billion spent on back and neck pain in 2016 $(15,16)$. Additionally, spending on back and neck pain, and musculoskeletal disorders moved to the number 1 and 2 categories. Despite these extensive expendi- tures, spinal pain disability continues as the number one cause of disability, whereas neck pain ranks as number 3 (17-21).

Recently developed guidance from the Department of Health and Human Services (HHS) on Best Practices in Pain Management prominently focuses on interventional techniques (20). The opioid epidemic, which was under control until 2018, has started increasing since 2019, with exploding patterns in 2020 due to COVID-19 (22-32). In fact, reduced access to interventional techniques has been considered as one of the reasons for increasing abuse patterns, as patients are sent to the street because of their inability to undergo interventional techniques and at the same time, an inability to receive appropriate opioid prescriptions (22-25). This has led to significant stress among physicians in 2020 (28). In addition, COVID-19 has also affected education and training $(30,31)$. More recently, many practices are being sold to the hospitals or other organizations causing the extinction of independent practices (33). COVID-19's storm and reduced access may cause further declines in utilization patterns as well as costs (22-32).

The utilization patterns of interventional techniques overall from 2000 to 2018 showed a decline of all interventional techniques at an annual rate of $0.8 \%$ with an overall decline of $6.7 \%$ (1). Specifically, epidural procedure utilization has declined at a rate of $20.7 \%$ per 100,000 Medicare enrollees from 2009 to 2018 , with an annual decline of $2.5 \%$. There were escalating increases in earlier years of $89.2 \%$, with an annual increase of $7.3 \%$ from 2000 to 2009 (2). This analysis specifically showed a decline in all categories, with an annual decrease of $4.7 \%$ for lumbar interlaminar and caudal epidural procedures, $4.7 \%$ decline for cervical and thoracic transforaminal epidural procedures, $1.1 \%$ decline for lumbosacral transforaminal epidural procedures, and finally, $0.4 \%$ decline for cervical and thoracic interlaminar epidural procedures. Thus, this analysis showed that from 2009 to 2018, the highest declines were noted for cervical and thoracic transforaminal epidural procedures with $35.1 \%$, closely followed by lumbar interlaminar and caudal epidural procedures of $34.9 \%$, and with a distant follow-up of $9.4 \%$ for lumbosacral transforaminal epidurals, and the least decline of $3.5 \%$ for cervical and thoracic interlaminar epidurals. This manuscript revealed interesting trends with 3 to 4 times higher increases of lumbar interlaminar and caudal epidural procedures compared to lumbosacral 
transforaminal epidurals, showing a reversal of the previous trend (2).

Despite the continued criticism along with declining utilization, cost utility analysis in favor of epidural procedures compared to multiple other modalities of treatments, has been published in numerous performed studies (34-43).

To date, there has not been a systematic assessment of the cost of epidural procedures since 2008 (14). In this manuscript, Manchikanti et al (14) assessed the growth of spinal interventional pain management techniques, along with Medicare expenditures from 2000 to 2008. However, in that analysis, the authors did not look at facet joint interventions or epidural procedures, and their costs separately. Recently, Manchikanti et al (44) assessed trends of expenditures and utilization patterns for facet joint interventions in the FFS Medicare population. This analysis showed that even after adjusting for inflation, there was a significant increase in the expenditures for facet joint interventions with an overall $53 \%$ increase. Inflation-adjusted cost per year declined $7 \%$ overall and $0.8 \%$ annually from $\$ 1,925$ to $\$ 1,785$, and inflation-adjusted cost per visit also declined $11 \%$ overall and $1.3 \%$ annually from $\$ 952$ in 2009 to $\$ 850$ in 2018.

This manuscript, therefore, was undertaken to assess expenditures and utilization patterns of epidural procedures from 2009 to 2018.

\section{Methods}

This analysis of expenditures and utilization patterns in the FFS Medicare population was performed utilizing a retrospective cohort analysis with methodology as described by the Strengthening and Reporting of Observational Studies in Epidemiology (STROBE) (45). The data was obtained from the Centers for Medicare and Medicaid Services' (CMS) physician outpatient billing claims for those enrolled in the FFS Medicare program for 2009 through 2018, consisting of the standard $5 \%$ national sample (46). The sample data consisting of $5 \%$ from CMS, has been reported to be unbiased and unpredictable to avoid divulging of any patient characteristics. However, the data does allow appropriate tracking of patients over time and across databases. Consequently, the Institutional Review Board (IRB) approval was not required.

\section{Study Design}

The estimation of expenditures for epidural pro- cedures in FFS Medicare recipients was designed as a retrospective cohort study calculating the trends of costs and utilization patterns from 2009 to 2018 in the United States (46). In this analysis:

- A patient was considered as undergoing epidural procedures throughout the year, irrespective of number of visits, episodes, or services.

- A visit included all regions treated during the visit.

- An episode was considered as one per region utilizing primary codes only.

- Services or procedures were considered as all procedures, multiple levels including add-on codes and bilaterals.

\section{Setting}

The standard $5 \%$ national sample data was obtained from the CMS services physician outpatient billing claims for those enrolled in the FFS Medicare program from 2009 to 2018. Participants included all Medicare FFS recipients receiving epidural procedures. The current procedural terminology (CPT) codes included in this analysis are listed in Table 1.

\section{Data Sources}

CMS physician outpatient billing claims for those enrolled in the FFS Medicare program from 2009 to 2018 provided the appropriate data, facilitating the analysis.

Table 1. CPT codes utilized for epidural procedures from 2009 to 2018.

\begin{tabular}{|l|l||}
\hline CPT Code & Description \\
\hline 64479 & Cervical/Thoracic Transforaminal Epidurals \\
\hline 64480 & $\begin{array}{l}\text { Cervical/Thoracic Transforaminal Epidurals } \\
\text { add-on }\end{array}$ \\
\hline 64483 & Lumbar/Sacral Transforaminal Epidurals \\
\hline 64484 & $\begin{array}{l}\text { Lumbar/Sacral Transforaminal Epidurals } \\
\text { add-on }\end{array}$ \\
\hline Codes Until 2016 \\
\hline 62310 & Cervical/Thoracic Interlaminar Epidurals \\
\hline 62311 & Lumbar Interlaminar and Caudal Epidurals \\
\hline Codes from 2017 to 2018 \\
\hline 62320 (62310) & $\begin{array}{l}\text { Cervical/Thoracic Interlaminar Epidurals } \\
\text { without fluoroscopy }\end{array}$ \\
\hline $62321(62310)$ & $\begin{array}{l}\text { Cervical/Thoracic Interlaminar Epidurals with } \\
\text { fluoroscopy }\end{array}$ \\
\hline 62322 (62311) & $\begin{array}{l}\text { Lumbar Interlaminar and Caudal Epidurals } \\
\text { without fluoroscopy }\end{array}$ \\
\hline 62323 (62311) & $\begin{array}{l}\text { Lumbar Interlaminar and Caudal Epidurals } \\
\text { with fluoroscopy }\end{array}$ \\
\hline
\end{tabular}




\section{Data Compilation}

Data was compiled utilizing Microsoft 365 Access and Microsoft 365 Excel (Microsoft, Redmond, WA). We removed all epidural procedure services with zero allowed payments. One hundred percent data was obtained by multiplication with 20 to scale up from our $5 \%$ sample to the full M-FFS population. The data were calculated for overall services for each procedure, and the rate of services, based on utilization per 100,000 FFS Medicare beneficiaries. Expenditures were also calculated for physician and facility, which included allowable charges for physician and facility (ambulatory surgery center [ASC], hospital outpatient department [HOPD], office setting). All the expenditures were presented with allowed costs and were adjusted for inflation to 2018 US dollars. HOPD facility allowed charges were estimated based on National Average rates.

\section{Variables}

The analysis of trends of utilization and costs patterns of epidural procedures incorporated multiple variables with analysis and costs for all procedures, utilization based on statewide and Medicare Administrative Contractors (MACs) and location of the service provided, either office-, ASC-, or HOPD-based.

\section{Measures}

Allowed services were assessed for each procedure. Rates were calculated based on Medicare beneficiaries for the corresponding year and are reported as procedures per 100,000 Medicare beneficiaries. Data was assessed for the total number of procedures performed, as well as the number of visits or sessions for epidural procedures. An episode is considered as one per region, irrespective of number of procedures performed.

\section{Bias}

Data was purchased from the CMS by the American Society of Interventional Pain Physicians (ASIPP). The study was conducted with the internal resources of the primary author's practice without external funding. The costs were determined without eliciting any bias. Thus, based on the large size of the dataset derived from a government source, there was no information related to patients' individual identification.

\section{Sample Size}

The size of this retrospective cohort study is robust, providing real-world claims data on Medicare patients with inclusion of all Medicare FFS patients undergoing epidural procedures for spinal pain from 2009 to 2018.

\section{Results}

\section{Participants and Characteristics}

In this analysis, the participants were from the Medicare database undergoing epidural procedures from 2009 to 2018.

\section{Utilization Characteristics}

Table 2 shows descriptive data of epidural procedures and population characteristics. Medicare beneficiaries grew at an annual rate of $3 \%$, whereas the US population grew at $0.7 \%$. Allowed epidural services were 2,118,840 in 2009, increasing to 2,196,100, an increase of $3.6 \%$, at a rate of $0.4 \%$, which is much lower than the growth rate of Medicare beneficiaries. Consequently, the rate of these procedures per 100,000 population decreased $20.4 \%$, with an annual decrease of $2.5 \%$ from 4,626 in 2009 to 3,685 in 2018 . The number of patients receiving epidural procedures increased in terms of absolute number from 856,540 in 2009 to $1,013,080$ in 2018 , with an overall increase of $18.3 \%$, and an annual increase of $1.9 \%$. However, the overall rate of procedures performed decreased by $9.1 \%$ and $1.1 \%$ annually with 1,870 per 100,000 Medicare population in 2009 to 1,700 in 2018. Figure 1 shows a graphic display of the rate of epidural procedures by services, episodes, and patients from 2009 to 2018 without increasing any of the aspects, except for decreases in services and rates.

Table 3 shows services and frequency of rates of epidural procedures. Interlaminar/caudal versus transforaminal showing significant differences, as shown in a previous manuscript (2), with an overall decrease of $33.3 \%$ and an annual decrease of $4.4 \%$ per 100,000 Medicare population. However, with a decrease in services of $6.5 \%$ and an annual decrease of $0.7 \%$ for transforaminal per 100,000 Medicare population. Figure 2 shows the results in a graphic format.

Appendix Table 1 shows the utilization patterns of epidural procedures by various specialty groups from 2009 to 2018 with overall interventional pain management groups, including interventional pain management, anesthesiology, physical medicine and rehabilitation (PMR), neurology, and pain management. Interventional pain management specialties including PMR, neurology, and psychiatry performed $90.4 \%$ of the total patient care with an increased rate of utili- 
Declining Expenditures for Epidural Procedures in the Medicare Population

Table 2. Characteristics of Medicare beneficiaries and the utilization pattern of epidural interventions from 2009 to 2018.

\begin{tabular}{|c|c|c|c|c|c|c|c|c|c|c|c|c|}
\hline & F2009 & F2010 & F2011 & F2012 & F2013 & F2014 & F2015 & F2016 & F2017 & F2018 & Change & Rate \\
\hline $\begin{array}{l}\text { US } \\
\text { Population }\end{array}$ & 307,006 & 308,746 & 311,583 & 313,874 & 316,129 & 318,892 & 320,897 & 323,127 & 326,625 & 327,167 & $6.6 \%$ & $0.7 \%$ \\
\hline$\geq 65$ years & 39,570 & 40,268 & 41,370 & 43,144 & 44,704 & 46,179 & 47,734 & 49,244 & 51,055 & 52,347 & $32.3 \%$ & $3.2 \%$ \\
\hline $\begin{array}{l}\text { Medicare } \\
\text { beneficiaries' }\end{array}$ & 45,801 & 46,914 & 48,300 & 50,300 & 51,900 & 53,500 & 54,900 & 56,500 & 58,000 & 59,600 & $30.1 \%$ & $3.0 \%$ \\
\hline$\geq 65$ years & 38,177 & 38,991 & 40,000 & 41,900 & 43,100 & 44,600 & 46,000 & 47,500 & 49,200 & 50,800 & $33.1 \%$ & $3.2 \%$ \\
\hline$\% \geq 65$ years & $83.4 \%$ & $83.1 \%$ & $82.8 \%$ & $83.1 \%$ & $83.0 \%$ & $83.4 \%$ & $83.6 \%$ & $84.1 \%$ & $84.7 \%$ & $85.2 \%$ & $2.3 \%$ & $0.2 \%$ \\
\hline$<65$ years & 7,624 & 7,923 & 8,300 & 8,500 & 8,800 & 8,900 & 9,000 & 9,000 & 8,900 & 8,800 & $15.4 \%$ & $1.6 \%$ \\
\hline $\begin{array}{l}\text { Epidural } \\
\text { Services }\end{array}$ & F2009 & F2010 & F2011 & F2012 & F2013 & F2014 & F2015 & F2016 & F2017 & F2018 & Change & Rate \\
\hline $\begin{array}{l}\text { Services } \\
\text { (Allowed) }\end{array}$ & $2,118,840$ & $2,205,160$ & $2,290,740$ & $2,311,880$ & $2,251,720$ & $2,268,300$ & $2,288,520$ & $2,335,000$ & $2,197,300$ & $2,196,100$ & $3.6 \%$ & $0.4 \%$ \\
\hline Rate & 4,626 & 4,700 & 4,743 & 4,596 & 4,339 & 4,240 & 4,169 & 4,133 & 3,788 & 3,685 & $-20.4 \%$ & $-2.5 \%$ \\
\hline Episodes & $1,727,640$ & $1,793,240$ & $1,866,800$ & $1,894,380$ & $1,849,100$ & $1,836,400$ & $1,851,940$ & $1,895,620$ & $1,785,900$ & $1,798,100$ & $4.1 \%$ & $0.4 \%$ \\
\hline Rate & 3,772 & 3,822 & 3,865 & 3,766 & 3,563 & 3,433 & 3,373 & 3,355 & 3,079 & 3,017 & $-20.0 \%$ & $-2.5 \%$ \\
\hline Visits & $1,681,200$ & $1,748,660$ & $1,823,380$ & $1,853,120$ & $1,831,420$ & $1,822,260$ & $1,842,720$ & $1,887,260$ & $1,778,580$ & $1,791,200$ & $6.5 \%$ & $0.7 \%$ \\
\hline Rate & 3,671 & 3,727 & 3,775 & 3,684 & 3,529 & 3,406 & 3,357 & 3,340 & 3,067 & 3,005 & $-18.1 \%$ & $-2.2 \%$ \\
\hline Patients & 856,540 & 891,640 & 936,500 & 967,080 & 959,520 & 971,280 & 993,960 & $1,027,120$ & $1,001,700$ & $1,013,080$ & $18.3 \%$ & $1.9 \%$ \\
\hline Rate & 1,870 & 1,901 & 1,939 & 1,923 & 1,849 & 1,815 & 1,810 & 1,818 & 1,727 & 1,700 & $-9.1 \%$ & $-1.1 \%$ \\
\hline \multicolumn{13}{|c|}{ Age groups (Patients) } \\
\hline$\geq 65$ Years & 686,060 & 711,020 & 737,080 & 756,680 & 747,640 & 760,140 & 783,140 & 820,060 & 809,940 & 832,000 & $21.3 \%$ & $2.2 \%$ \\
\hline$\%$ & $80.1 \%$ & $79.7 \%$ & $78.7 \%$ & $78.2 \%$ & $77.9 \%$ & $78.3 \%$ & $78.8 \%$ & $79.8 \%$ & $80.9 \%$ & $82.1 \%$ & $2.5 \%$ & $0.3 \%$ \\
\hline Rate & 1,498 & 1,516 & 1,526 & 1,504 & 1,441 & 1,421 & 1,426 & 1,451 & 1,396 & 1,396 & $-6.8 \%$ & $-0.8 \%$ \\
\hline$<65$ Years & 170,480 & 180,620 & 199,420 & 210,400 & 211,880 & 211,140 & 210,820 & 207,060 & 191,760 & 181,080 & $6.2 \%$ & $0.7 \%$ \\
\hline Rate & 372 & 385 & 413 & 418 & 408 & 395 & 384 & 366 & 331 & 304 & $-18.4 \%$ & $-2.2 \%$ \\
\hline \multicolumn{13}{|c|}{ Episodes by age } \\
\hline$\geq 65$ & $1,365,840$ & $1,413,080$ & $1,452,280$ & $1,466,500$ & $1,421,500$ & $1,421,960$ & $1,446,800$ & $1,501,960$ & $1,433,840$ & $1,466,960$ & $7.4 \%$ & $0.8 \%$ \\
\hline Rate & 2,982 & 3,012 & 3,007 & 2,916 & 2,739 & 2,658 & 2,635 & 2,658 & 2,472 & 2,461 & $-17.5 \%$ & $-2.1 \%$ \\
\hline$<65$ & 361,800 & 380,160 & 414,520 & 427,880 & 427,600 & 414,440 & 405,140 & 393,660 & 352,060 & 331,140 & $-8.5 \%$ & $-1.0 \%$ \\
\hline Rate & 790 & 810 & 858 & 851 & 824 & 775 & 738 & 697 & 607 & 556 & $-29.7 \%$ & $-3.8 \%$ \\
\hline \multicolumn{13}{|c|}{ Episodes by PLCR } \\
\hline HOPD & 577,100 & 591,640 & 618,400 & 611,780 & 586,380 & 584,120 & 581,020 & 587,380 & 538,880 & 538,200 & $-6.7 \%$ & $-0.8 \%$ \\
\hline Rate & 1,260 & 1,261 & 1,280 & 1,216 & 1,130 & 1,092 & 1,058 & 1,040 & 929 & 903 & $-28.3 \%$ & $-3.6 \%$ \\
\hline ASC & 460,740 & 469,840 & 501,920 & 522,560 & 498,040 & 502,180 & 511,920 & 542,800 & 508,100 & 510,360 & $10.8 \%$ & $1.1 \%$ \\
\hline Rate & 1,006 & 1,001 & 1,039 & 1,039 & 960 & 939 & 932 & 961 & 876 & 856 & $-14.9 \%$ & $-1.8 \%$ \\
\hline Office & 689,800 & 731,760 & 746,480 & 760,040 & 764,680 & 750,100 & 759,000 & 765,440 & 738,920 & 749,540 & $8.7 \%$ & $0.9 \%$ \\
\hline Rate & 1,506 & 1,560 & 1,546 & 1,511 & 1,473 & 1,402 & 1,383 & 1,355 & 1,274 & 1,258 & $-16.5 \%$ & $-2.0 \%$ \\
\hline
\end{tabular}

Rate: per 100,000 Medicare beneficiaries; Change: of change from 2009 to 2018, GM - geometric average. PCPY - percentage of change from previous year

zation among these groups. In contrast, surgical groups, radiology groups, and other providers showed a decline of utilization patterns.

Appendix Table 2 shows the utilization of epidural procedures per 100,000 Medicare population, with declines observed in almost all jurisdictions, with an average decline across the United States of $20 \%$, and an annual decline of $2.5 \%$.

For comparative purposes, Appendix Table 3 shows the utilization of lumbar interlaminar and caudal epidural procedure rates in the Medicare population, with declines in all jurisdictions. 
Similarly, Appendix Table 4 shows the utilization patterns of lumbar transforaminal epidural procedures, which decreased at a lesser rate than interlaminar epidural procedures, $33.3 \%$ versus $6.5 \%$, and $4.4 \%$ annual compared to $0.7 \%$ annual.

\section{Expenditure Characteristics}

Table 4 shows the average allowed charges per service or procedure, which showed, with inflation- adjusted, a decline of $5.5 \%$ or $0.6 \%$ annually. However, prior to inflation-adjustment, the average allowed charges increased $10.6 \%$ and $1.1 \%$ from $\$ 341.69$ in 2009 to $\$ 377.94$ in 2018. With inflation-adjustment, they were $\$ 399.77$ to $\$ 377.94$.

Table 5 shows the average allowed charges per visit, which includes multiple regions only, but not other interventions, which declined after inflationadjustment by $8 \%$ and $0.9 \%$ from $\$ 503.84$ in 2009 to $\$ 463.37$ in 2018; however, prior to inflation-adjustment, they increased by $7.6 \%$ and an annual increase of $0.8 \%$ from $\$ 430.63$ to $\$ 463.37$.

Table 6 shows the average allowed charges per patient, with an average of $\$ 845.24$ to $\$ 819.27$ in 2018 , with a decline of $3.1 \%$ and $0.3 \%$ per year prior to inflation-adjustment. However, the inflationadjusted rate showed even steeper declines from $\$ 988.93$ to $\$ 819.27$, a $17.2 \%$ decrease with $0.21 \%$ decrease annually.

Table 7 shows the total allowed charges by place of service and type of procedure, which included all services

Fig. 1. Epidural procedures rate per 100,000 FFS Medicare population by services, episodes, and patients from 2009-2018.

Table 3. Services and frequency of rates for epidural procedures (interlaminar/caudal vs. transforaminal) in the Medicare population from 2009 to 2018.

\begin{tabular}{|c|c|c|c|c|c|c|c|c|c|c|c|c|c|c|}
\hline & \multicolumn{2}{|c|}{$\begin{array}{l}\text { C/T Interlaminar } \\
\text { Epidurals } \\
\text { (CPT 62310/62321) }\end{array}$} & \multicolumn{2}{|c|}{$\begin{array}{l}\mathrm{C} / \mathrm{T} \\
\text { Transforaminal } \\
\text { Epidurals } \\
\text { (CPT 94479) }\end{array}$} & \multirow[b]{2}{*}{ Ratio } & \multicolumn{2}{|c|}{$\begin{array}{l}\mathrm{C} / \mathrm{T} \\
\text { Transforaminal } \\
\text { Epidurals } \\
(64479-64480)\end{array}$} & \multicolumn{2}{|c|}{$\begin{array}{l}\text { Lumbar } \\
\text { Interlaminar and } \\
\text { Caudal Epidurals } \\
\text { (CPT 62311) }\end{array}$} & \multicolumn{2}{|c|}{$\begin{array}{l}\text { Lumbar } \\
\text { Transforaminal } \\
\text { Epidurals } \\
(64483)\end{array}$} & \multirow[b]{2}{*}{ Ratio } & \multicolumn{2}{|c|}{$\begin{array}{l}\text { Lumbar } \\
\text { Transforaminal } \\
\text { Epidurals } \\
(64483-64484)\end{array}$} \\
\hline & Services & Rate & 64479 & Rate & & Services & Rate & 62311 & Rate & 64483 & Rate & & Services & Rate \\
\hline F2009 & 174,240 & 380 & 38,120 & 83 & 4.6 & 68,120 & 149 & 876,580 & 1,914 & 638,700 & 1,395 & 1.4 & 999,900 & 2,183 \\
\hline F2010 & 182,240 & 388 & 41,960 & 89 & 4.3 & 74,340 & 158 & 885,520 & 1,888 & 683,520 & 1,457 & 1.3 & $1,063,060$ & 2,266 \\
\hline F2011 & 199,700 & 413 & 38,780 & 80 & 5.1 & 65,580 & 136 & 912,520 & 1,889 & 715,800 & 1,482 & 1.3 & $1,112,940$ & 2,304 \\
\hline F2012 & 209,940 & 417 & 35,780 & 71 & 5.9 & 56,840 & 113 & 921,120 & 1,831 & 727,540 & 1,446 & 1.3 & $1,123,980$ & 2,235 \\
\hline F2013 & 215,500 & 415 & 34,360 & 66 & 6.3 & 54,240 & 105 & 896,880 & 1,728 & 702,360 & 1,353 & 1.3 & $1,085,100$ & 2,091 \\
\hline F2014 & 208,140 & 389 & 38,900 & 73 & 5.4 & 60,320 & 113 & 819,300 & 1,531 & 770,060 & 1,439 & 1.1 & $1,180,540$ & 2,207 \\
\hline F2015 & 218,560 & 398 & 39,120 & 71 & 5.6 & 60,560 & 110 & 817,300 & 1,489 & 776,960 & 1,415 & 1.1 & $1,192,100$ & 2,171 \\
\hline F2016 & 224,480 & 397 & 39,940 & 71 & 5.6 & 60,760 & 108 & 829,640 & 1,468 & 801,560 & 1,419 & 1.0 & $1,220,120$ & 2,160 \\
\hline F2017 & 211,520 & 365 & 38,720 & 67 & 5.5 & 57,180 & 99 & 754,040 & 1,300 & 781,620 & 1,348 & 1.0 & $1,174,560$ & 2,025 \\
\hline F2018 & 221,560 & 372 & 38,260 & 64 & 5.8 & 55,580 & 93 & 760,920 & 1,277 & 777,360 & 1,304 & 1.0 & $1,158,040$ & 1,943 \\
\hline Change & $27.2 \%$ & $-2.3 \%$ & $0.4 \%$ & $-22.9 \%$ & & $-18.4 \%$ & $-37.3 \%$ & $-13.2 \%$ & $-33.3 \%$ & $21.7 \%$ & $-6.5 \%$ & & $15.8 \%$ & $-11.0 \%$ \\
\hline GM & $2.7 \%$ & $-0.3 \%$ & $0.0 \%$ & $-2.8 \%$ & & $-2.2 \%$ & $-5.1 \%$ & $-1.6 \%$ & $-4.4 \%$ & $2.2 \%$ & $-0.7 \%$ & & $1.6 \%$ & $-1.3 \%$ \\
\hline
\end{tabular}

Rate: per 100,000 Medicare beneficiaries; Change: of change from 2009 to 2018; GM - geometric average. 
Declining Expenditures for Epidural Procedures in the Medicare Population

and locations. Overall, the costs increased $4.6 \%$ and $1.5 \%$ from $\$ 723,981,554$ to $\$ 829,987,636$. After inflation-adjustment, they declined $2 \%$ or $0.2 \%$ annually from $\$ 847,058,465$ to $\$ 829,987,636$. Overall, costs per procedure in 2018 were $\$ 618.79$ in HOPD settings, which essentially recorded an increase of $21.3 \%$ and $2.2 \%$, compared to $\$ 381.59$ in an ASC setting, with an increase of $1 \%$ and $0.1 \%$, compared to $\$ 218.23$ with an increase of $13.5 \%$ and $1.4 \%$ annually. Similarly, allowed charges per patient were also higher in HOPD, followed by ASC, then followed by office. Further, the average allowed charges per

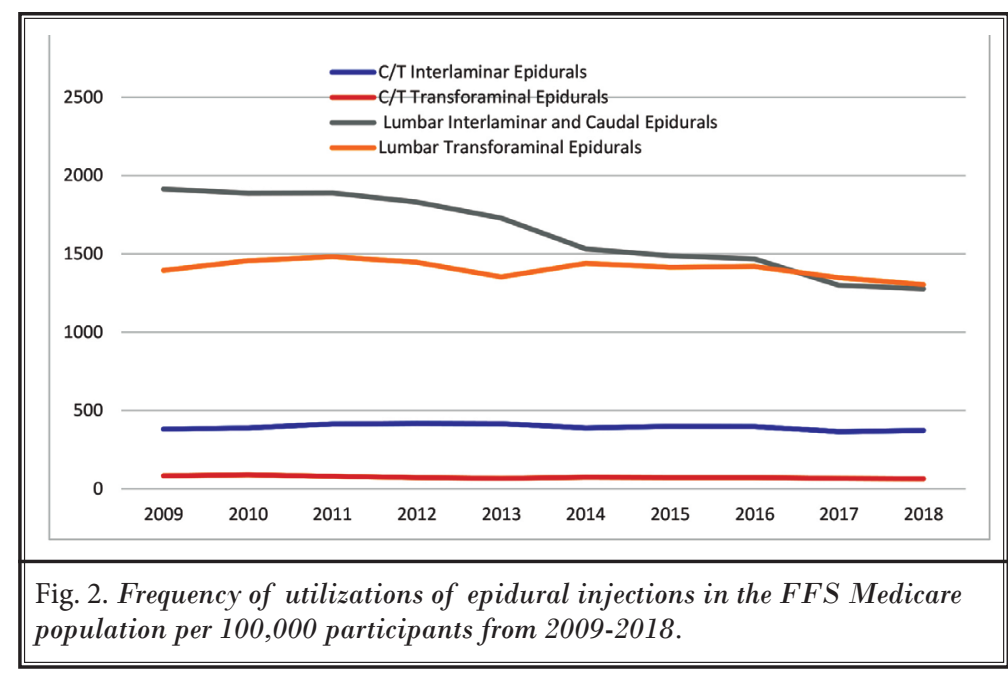

Table 4. Average allowed charges per service or procedure.

\begin{tabular}{|c|c|c|c|c|c|c|c|c|c|c|c|c|}
\hline PLCR & 2009 & 2010 & 2011 & 2012 & 2013 & 2014 & 2015 & 2016 & 2017 & 2018 & Change & GM \\
\hline HOPD & 661,140 & 673,200 & 708,800 & 698,260 & 669,380 & 670,060 & 667,320 & 677,420 & 618,560 & 616,560 & $-6.7 \%$ & $-0.8 \%$ \\
\hline ASC & 572,900 & 585,860 & 628,860 & 660,200 & 623,960 & 627,160 & 642,240 & 675,660 & 638,380 & 635,180 & $10.9 \%$ & $1.2 \%$ \\
\hline Office & 884,800 & 946,100 & 953,080 & 953,420 & 958,380 & 971,080 & 978,960 & 981,920 & 940,360 & 944,360 & $6.7 \%$ & $0.7 \%$ \\
\hline Total & $2,118,840$ & $2,205,160$ & $2,290,740$ & $2,311,880$ & $2,251,720$ & $2,268,300$ & $2,288,520$ & $2,335,000$ & $2,197,300$ & $2,196,100$ & $3.6 \%$ & $0.4 \%$ \\
\hline \multicolumn{13}{|l|}{ Average } \\
\hline \multicolumn{13}{|c|}{ Professional } \\
\hline HOPD & $\$ 84.45$ & $\$ 89.62$ & $\$ 88.89$ & $\$ 92.93$ & $\$ 96.30$ & $\$ 85.79$ & $\$ 98.91$ & $\$ 98.89$ & $\$ 104.09$ & $\$ 103.85$ & $23.0 \%$ & $2.3 \%$ \\
\hline ASC & $\$ 86.72$ & $\$ 92.85$ & $\$ 90.82$ & $\$ 95.08$ & $\$ 98.96$ & $\$ 91.87$ & $\$ 102.17$ & $\$ 102.85$ & $\$ 106.05$ & $\$ 106.49$ & $22.8 \%$ & $2.3 \%$ \\
\hline Office & $\$ 85.59$ & $\$ 92.73$ & $\$ 90.60$ & $\$ 95.07$ & $\$ 97.76$ & $\$ 92.94$ & $\$ 100.77$ & $\$ 101.39$ & $\$ 103.68$ & $\$ 104.81$ & $22.5 \%$ & $2.3 \%$ \\
\hline Total & $\$ 85.54$ & $\$ 91.81$ & $\$ 90.13$ & $\$ 94.43$ & $\$ 97.66$ & $\$ 90.53$ & $\$ 100.62$ & $\$ 101.09$ & $\$ 104.48$ & $\$ 105.02$ & $22.8 \%$ & $2.3 \%$ \\
\hline \multicolumn{13}{|l|}{ Facility } \\
\hline HOPD & $\$ 425.85$ & $\$ 430.99$ & $\$ 473.06$ & $\$ 474.05$ & $\$ 513.69$ & $\$ 583.99$ & $\$ 584.92$ & $\$ 507.39$ & $\$ 483.78$ & $\$ 514.94$ & $20.9 \%$ & $2.1 \%$ \\
\hline ASC & $\$ 291.07$ & $\$ 281.43$ & $\$ 273.64$ & $\$ 273.29$ & $\$ 288.16$ & $\$ 316.09$ & $\$ 316.18$ & $\$ 282.22$ & $\$ 268.82$ & $\$ 275.11$ & $-5.5 \%$ & $-0.6 \%$ \\
\hline Office & $\$ 106.73$ & $\$ 112.73$ & $\$ 114.23$ & $\$ 111.60$ & $\$ 110.50$ & $\$ 70.33$ & $\$ 105.46$ & $\$ 106.34$ & $\$ 109.80$ & $\$ 113.42$ & $6.3 \%$ & $0.7 \%$ \\
\hline Total & $\$ 256.15$ & $\$ 254.71$ & $\$ 269.02$ & $\$ 267.24$ & $\$ 279.59$ & $\$ 290.02$ & $\$ 304.41$ & $\$ 273.59$ & $\$ 261.28$ & $\$ 272.91$ & $6.5 \%$ & $0.7 \%$ \\
\hline \multicolumn{13}{|c|}{ Total (Professional + Facility) } \\
\hline HOPD & $\$ 510.30$ & $\$ 520.60$ & $\$ 561.95$ & $\$ 566.98$ & $\$ 609.99$ & $\$ 669.78$ & $\$ 683.83$ & $\$ 606.28$ & $\$ 587.87$ & $\$ 618.79$ & $21.3 \%$ & $2.2 \%$ \\
\hline ASC & $\$ 377.79$ & $\$ 374.28$ & $\$ 364.47$ & $\$ 368.37$ & $\$ 387.12$ & $\$ 407.96$ & $\$ 418.36$ & $\$ 385.08$ & $\$ 374.87$ & $\$ 381.59$ & $1.0 \%$ & $0.1 \%$ \\
\hline Office & $\$ 192.32$ & $\$ 205.46$ & $\$ 204.83$ & $\$ 206.67$ & $\$ 208.25$ & $\$ 163.28$ & $\$ 206.24$ & $\$ 207.74$ & $\$ 213.49$ & $\$ 218.23$ & $13.5 \%$ & $1.4 \%$ \\
\hline Total & $\$ 341.69$ & $\$ 346.52$ & $\$ 359.15$ & $\$ 361.67$ & $\$ 377.24$ & $\$ 380.55$ & $\$ 405.03$ & $\$ 374.68$ & $\$ 365.76$ & $\$ 377.94$ & $10.6 \%$ & $1.1 \%$ \\
\hline PCPY & & $1.4 \%$ & $3.6 \%$ & $0.7 \%$ & $4.3 \%$ & $0.9 \%$ & $6.4 \%$ & $-7.5 \%$ & $-2.4 \%$ & $3.3 \%$ & & \\
\hline $\begin{array}{l}\text { Inflation } \\
\text { Rate }\end{array}$ & 1.17 & 1.15 & 1.12 & 1.09 & 1.08 & 1.06 & 1.06 & 1.05 & 1.02 & 1 & $-14.5 \%$ & $-1.7 \%$ \\
\hline $\begin{array}{l}\text { Total }^{*} \\
\text { (infl) }\end{array}$ & $\$ 399.77$ & $\$ 398.50$ & $\$ 402.25$ & $\$ 394.22$ & $\$ 407.42$ & $\$ 403.38$ & $\$ 429.33$ & $\$ 393.41$ & $\$ 373.08$ & $\$ 377.94$ & $-5.5 \%$ & $-0.6 \%$ \\
\hline PCPY & & $-0.3 \%$ & $0.9 \%$ & $-2.0 \%$ & $3.3 \%$ & $-1.0 \%$ & $6.4 \%$ & $-8.4 \%$ & $-5.2 \%$ & $1.3 \%$ & & \\
\hline
\end{tabular}

${ }^{*}$ Inflation-adjusted and converted to the year 2018 values. Change: of change from 2009 to 2018; GM - geometric average PCPY - percentage of change from previous year

Note: There was about a 16\% reduction in payment rates for C/T/L epidural injection in ASC \& HOPD settings in 2016 \& 2018. In 2014, payments for ASC \& HOPD primary codes increased and removed payments for add-on codes. 
Pain Physician: January/February 2021 24:1-15

Table 5. Average allowed charges per visit.

\begin{tabular}{|c|c|c|c|c|c|c|c|c|c|c|c|c|}
\hline PLCR & 2009 & 2010 & 2011 & 2012 & 2013 & 2014 & 2015 & 2016 & 2017 & 2018 & Change & GM \\
\hline \multicolumn{13}{|c|}{ No. of Visits } \\
\hline HOPD & 566,460 & 581,040 & 607,280 & 601,380 & 580,200 & 578,740 & 577,500 & 583,880 & 536,380 & 534,880 & $-5.6 \%$ & $-0.6 \%$ \\
\hline ASC & 445,040 & 457,520 & 488,380 & 509,180 & 493,140 & 498,020 & 508,860 & 539,480 & 505,220 & 507,960 & $14.1 \%$ & $1.5 \%$ \\
\hline Office & 669,700 & 710,080 & 727,720 & 742,560 & 758,080 & 745,500 & 756,360 & 763,900 & 736,980 & 748,360 & $11.7 \%$ & $1.2 \%$ \\
\hline Total & $1,681,200$ & $1,748,640$ & $1,823,380$ & $1,853,120$ & $1,831,420$ & $1,822,260$ & $1,842,720$ & $1,887,260$ & $1,778,580$ & $1,791,200$ & $6.5 \%$ & $0.7 \%$ \\
\hline \multicolumn{13}{|l|}{ Average } \\
\hline \multicolumn{13}{|c|}{ Professional } \\
\hline HOPD & $\$ 98.57$ & $\$ 103.83$ & $\$ 103.75$ & $\$ 107.91$ & $\$ 111.10$ & $\$ 99.33$ & $\$ 114.29$ & $\$ 114.73$ & $\$ 120.04$ & $\$ 119.71$ & $21.4 \%$ & $2.2 \%$ \\
\hline ASC & $\$ 111.63$ & $\$ 118.90$ & $\$ 116.95$ & $\$ 123.28$ & $\$ 125.21$ & $\$ 115.69$ & $\$ 128.95$ & $\$ 128.82$ & $\$ 134.00$ & $\$ 133.15$ & $19.3 \%$ & $2.0 \%$ \\
\hline Office & $\$ 113.08$ & $\$ 123.55$ & $\$ 118.66$ & $\$ 122.07$ & $\$ 123.59$ & $\$ 121.07$ & $\$ 130.43$ & $\$ 130.33$ & $\$ 132.29$ & $\$ 132.26$ & $17.0 \%$ & $1.8 \%$ \\
\hline Total & $\$ 107.81$ & $\$ 115.78$ & $\$ 113.23$ & $\$ 117.81$ & $\$ 120.07$ & $\$ 112.69$ & $\$ 124.96$ & $\$ 125.07$ & $\$ 129.08$ & $\$ 128.76$ & $19.4 \%$ & $2.0 \%$ \\
\hline \multicolumn{13}{|l|}{ Facility } \\
\hline HOPD & $\$ 497.03$ & $\$ 499.35$ & $\$ 552.14$ & $\$ 550.41$ & $\$ 592.64$ & $\$ 676.14$ & $\$ 675.89$ & $\$ 588.68$ & $\$ 557.90$ & $\$ 593.57$ & $19.4 \%$ & $2.0 \%$ \\
\hline ASC & $\$ 374.70$ & $\$ 360.37$ & $\$ 352.35$ & $\$ 354.34$ & $\$ 364.61$ & $\$ 398.05$ & $\$ 399.06$ & $\$ 353.47$ & $\$ 339.67$ & $\$ 344.01$ & $-8.2 \%$ & $-0.9 \%$ \\
\hline Office & $\$ 141.02$ & $\$ 150.20$ & $\$ 149.60$ & $\$ 143.29$ & $\$ 139.69$ & $\$ 91.61$ & $\$ 136.50$ & $\$ 136.69$ & $\$ 140.11$ & $\$ 143.13$ & $1.5 \%$ & $0.2 \%$ \\
\hline Total & $\$ 322.83$ & $\$ 321.20$ & $\$ 337.97$ & $\$ 333.40$ & $\$ 343.75$ & $\$ 361.00$ & $\$ 378.05$ & $\$ 338.49$ & $\$ 322.79$ & $\$ 334.60$ & $3.6 \%$ & $0.4 \%$ \\
\hline \multicolumn{13}{|c|}{ Total (Professional + Facility) } \\
\hline HOPD & $\$ 595.59$ & $\$ 603.18$ & $\$ 655.89$ & $\$ 658.32$ & $\$ 703.75$ & $\$ 775.46$ & $\$ 790.19$ & $\$ 703.41$ & $\$ 677.94$ & $\$ 713.28$ & $19.8 \%$ & $2.0 \%$ \\
\hline ASC & $\$ 486.33$ & $\$ 479.27$ & $\$ 469.30$ & $\$ 477.62$ & $\$ 489.81$ & $\$ 513.74$ & $\$ 528.01$ & $\$ 482.28$ & $\$ 473.67$ & $\$ 477.16$ & $-1.9 \%$ & $-0.2 \%$ \\
\hline Office & $\$ 254.09$ & $\$ 273.75$ & $\$ 268.26$ & $\$ 265.35$ & $\$ 263.28$ & $\$ 212.68$ & $\$ 266.93$ & $\$ 267.02$ & $\$ 272.40$ & $\$ 275.38$ & $8.4 \%$ & $0.9 \%$ \\
\hline Total & $\$ 430.63$ & $\$ 436.99$ & $\$ 451.21$ & $\$ 451.21$ & $\$ 463.82$ & $\$ 473.70$ & $\$ 503.01$ & $\$ 463.57$ & $\$ 451.87$ & $\$ 463.37$ & $7.6 \%$ & $0.8 \%$ \\
\hline PCPY & & $1.5 \%$ & $3.3 \%$ & $0.0 \%$ & $2.8 \%$ & $2.1 \%$ & $6.2 \%$ & $-7.8 \%$ & $-2.5 \%$ & $2.5 \%$ & & \\
\hline $\begin{array}{l}\text { Inflation } \\
\text { Rate }\end{array}$ & 1.17 & 1.15 & 1.12 & 1.09 & 1.08 & 1.06 & 1.06 & 1.05 & 1.02 & 1 & $-14.5 \%$ & $-1.7 \%$ \\
\hline $\begin{array}{l}\text { Total }^{*} \\
\text { (infl) }\end{array}$ & $\$ 503.84$ & $\$ 502.53$ & $\$ 505.35$ & $\$ 491.81$ & $\$ 500.92$ & $\$ 502.12$ & $\$ 533.19$ & $\$ 486.74$ & $\$ 460.91$ & $\$ 463.37$ & $-8.0 \%$ & $-0.9 \%$ \\
\hline PCPY & & $-0.3 \%$ & $0.6 \%$ & $-2.7 \%$ & $1.9 \%$ & $0.2 \%$ & $6.2 \%$ & $-8.7 \%$ & $-5.3 \%$ & $0.5 \%$ & & \\
\hline
\end{tabular}

*Inflation-adjusted and converted to the year 2018 values. Change: of change from 2009 to 2018; GM - geometric average; PCPY - percentage of change from previous year

Note: There was about a 16\% reduction in payment rates for C/T/L epidural injection in ASC \& HOPD settings in 2016 \& 2018. In 2014, payments for ASC \& HOPD primary codes increased and removed payments for add-on codes.

patient were $\$ 1,174.20$ in HOPD setting with a record increase of $8.9 \%$ and $1 \%$, compared to ASC at $\$ 834.87$ with a decline of $11.8 \%$ and $1.4 \%$, and in an office setting of $\$ 518.01$, with an overall decline of $4.1 \%$ and an annual decline of $0.5 \%$.

In reference to overall expenditures, based on the usual procedures for caudal epidural and lumbar interlaminar epidural, there were declines of $2.7 \%$ overall and $0.3 \%$ annually. In contrast, for lumbar transforaminal epidural procedures, the expenses were higher with a $27 \%$ increase and an annual increase of $2.7 \%$.

\section{Discussion}

The estimated costs and utilization patterns of epidural procedures from 2009 to 2018 in the Medicare FFS population shows an overall decline in utilization patterns per 100,000 Medicare population, along with inflation-adjusted reductions in the costs, except for transforaminal epidural procedures and low utilized procedures in cervical spine. The number of patients receiving epidural procedures per 100,000 Medicare population declined from 1,870 to 1,700 , a $9.1 \%$ decline, with an annual decline of $1.1 \%$. At the same time, the Medicare beneficiaries increased $30.1 \%$ or $3 \%$ annually. Thus, there is a net decline of epidural procedures based on population increases or per 100,000 Medicare population. The $80 \%$ of the patients receiving epidural procedures in 2009 and $82 \%$ in 2018 were above the 
Declining Expenditures for Epidural Procedures in the Medicare Population

Table 6. Average allowed annual charges per patient.

\begin{tabular}{|c|c|c|c|c|c|c|c|c|c|c|c|c|}
\hline PLCR & 2009 & 2010 & 2011 & 2012 & 2013 & 2014 & 2015 & 2016 & 2017 & 2018 & Change & GM \\
\hline \multicolumn{13}{|c|}{ No. of Visits } \\
\hline HOPD & 312,980 & 319,280 & 334,440 & 334,880 & 326,880 & 332,300 & 334,200 & 344,000 & 325,920 & 324,920 & $3.8 \%$ & $0.4 \%$ \\
\hline ASC & 228,680 & 237,500 & 253,040 & 268,460 & 261,960 & 267,260 & 276,860 & 295,020 & 287,400 & 290,320 & $27.0 \%$ & $2.7 \%$ \\
\hline Office & 314,880 & 334,860 & 349,020 & 363,740 & 370,680 & 371,720 & 382,900 & 388,100 & 388,380 & 397,840 & $26.3 \%$ & $2.6 \%$ \\
\hline Total & 856,540 & 891,640 & 936,500 & 967,080 & 959,520 & 971,280 & 993,960 & $1,027,120$ & $1,001,700$ & $1,013,080$ & $18.3 \%$ & $1.9 \%$ \\
\hline \multicolumn{13}{|l|}{ Average } \\
\hline \multicolumn{13}{|c|}{ Professional } \\
\hline HOPD & $\$ 178.40$ & $\$ 188.96$ & $\$ 188.39$ & $\$ 193.78$ & $\$ 197.20$ & $\$ 172.99$ & $\$ 197.50$ & $\$ 194.74$ & $\$ 197.55$ & $\$ 197.06$ & $10.5 \%$ & $1.1 \%$ \\
\hline ASC & $\$ 217.25$ & $\$ 229.04$ & $\$ 225.72$ & $\$ 233.83$ & $\$ 235.70$ & $\$ 215.59$ & $\$ 237.01$ & $\$ 235.56$ & $\$ 235.56$ & $\$ 232.98$ & $7.2 \%$ & $0.8 \%$ \\
\hline Office & $\$ 240.50$ & $\$ 261.99$ & $\$ 247.40$ & $\$ 249.19$ & $\$ 252.75$ & $\$ 242.81$ & $\$ 257.65$ & $\$ 256.53$ & $\$ 251.04$ & $\$ 248.78$ & $3.4 \%$ & $0.4 \%$ \\
\hline Total & $\$ 211.60$ & $\$ 227.06$ & $\$ 220.47$ & $\$ 225.74$ & $\$ 229.17$ & $\$ 211.43$ & $\$ 231.67$ & $\$ 229.81$ & $\$ 229.19$ & $\$ 227.67$ & $7.6 \%$ & $0.8 \%$ \\
\hline \multicolumn{13}{|l|}{ Facility } \\
\hline HOPD & $\$ 899.56$ & $\$ 908.73$ & $\$ 1,002.59$ & $\$ 988.44$ & $\$ 1,051.92$ & $\$ 1,177.57$ & $\$ 1,167.95$ & $\$ 999.18$ & $\$ 918.16$ & $\$ 977.13$ & $8.6 \%$ & $0.9 \%$ \\
\hline ASC & $\$ 729.21$ & $\$ 694.23$ & $\$ 680.06$ & $\$ 672.07$ & $\$ 686.37$ & $\$ 741.74$ & $\$ 733.46$ & $\$ 646.36$ & $\$ 597.11$ & $\$ 601.90$ & $-17.5 \%$ & $-2.1 \%$ \\
\hline Office & $\$ 299.92$ & $\$ 318.50$ & $\$ 311.92$ & $\$ 292.51$ & $\$ 285.68$ & $\$ 183.74$ & $\$ 269.63$ & $\$ 269.05$ & $\$ 265.86$ & $\$ 269.23$ & $-10.2 \%$ & $-1.2 \%$ \\
\hline Total & $\$ 633.64$ & $\$ 629.93$ & $\$ 658.04$ & $\$ 638.86$ & $\$ 656.11$ & $\$ 677.29$ & $\$ 700.87$ & $\$ 621.96$ & $\$ 573.14$ & $\$ 591.61$ & $-6.6 \%$ & $-0.8 \%$ \\
\hline \multicolumn{13}{|c|}{ Total (Professional + Facility) } \\
\hline HOPD & $\$ 1,077.96$ & $\$ 1,097.69$ & $\$ 1,190.98$ & $\$ 1,182.22$ & $\$ 1,249.12$ & $\$ 1,350.56$ & $\$ 1,365.45$ & $\$ 1,193.92$ & $\$ 1,115.71$ & $\$ 1,174.20$ & $8.9 \%$ & $1.0 \%$ \\
\hline ASC & $\$ 946.46$ & $\$ 923.27$ & $\$ 905.78$ & $\$ 905.90$ & $\$ 922.07$ & $\$ 957.32$ & $\$ 970.47$ & $\$ 881.91$ & $\$ 832.67$ & $\$ 834.87$ & $-11.8 \%$ & $-1.4 \%$ \\
\hline Office & $\$ 540.41$ & $\$ 580.49$ & $\$ 559.33$ & $\$ 541.71$ & $\$ 538.43$ & $\$ 426.54$ & $\$ 527.28$ & $\$ 525.58$ & $\$ 516.90$ & $\$ 518.01$ & $-4.1 \%$ & $-0.5 \%$ \\
\hline Total & $\$ 845.24$ & $\$ 856.99$ & $\$ 878.51$ & $\$ 864.60$ & $\$ 885.28$ & $\$ 888.72$ & $\$ 932.55$ & $\$ 851.77$ & $\$ 802.33$ & $\$ 819.27$ & $-3.1 \%$ & $-0.3 \%$ \\
\hline PCPY & & $1.4 \%$ & $2.5 \%$ & $-1.6 \%$ & $2.4 \%$ & $0.4 \%$ & $4.9 \%$ & $-8.7 \%$ & $-5.8 \%$ & $2.1 \%$ & & \\
\hline $\begin{array}{l}\text { Inflation } \\
\text { Rate }\end{array}$ & 1.17 & 1.15 & 1.12 & 1.09 & 1.08 & 1.06 & 1.06 & 1.05 & 1.02 & 1 & $-14.5 \%$ & $-1.7 \%$ \\
\hline $\begin{array}{l}\text { Total }{ }^{*} \\
\text { (infl) }\end{array}$ & $\$ 988.93$ & $\$ 985.54$ & $\$ 983.93$ & $\$ 942.41$ & $\$ 956.10$ & $\$ 942.05$ & $\$ 988.50$ & $\$ 894.36$ & $\$ 818.38$ & $\$ 819.27$ & $-17.2 \%$ & $-2.1 \%$ \\
\hline PCPY & & $-0.3 \%$ & $-0.2 \%$ & $-4.2 \%$ & $1.5 \%$ & $-1.5 \%$ & $4.9 \%$ & $-9.5 \%$ & $-8.5 \%$ & $0.1 \%$ & & \\
\hline
\end{tabular}

${ }^{\star}$ Inflation-adjusted and converted to the year 2018 values. Change: of change from 2009 to 2018; GM - geometric average; PCPY - percentage of change from previous year

Note: There was about a 16\% reduction in payment rates for C/T/L epidural injection in ASC \& HOPD settings in 2016 \& 2018. In 2014, payments for ASC \& HOPD primary codes increased and removed payments for add-on codes.

age of 65 years, whereas the remaining were younger disabled individuals. The proportion of patient episodes in various settings was 1,258 per 100,000 Medicare population in an office setting, 903 in an HOPD setting, and 856 in an ASC. The data also showed consistently substantial declines of interlaminar epidural procedures compared to very mild decreases for lumbar transforaminal epidural procedures, which essentially is considered as an increase.

As shown in Table 4, average allowed charges per service or procedure after inflation-adjustment decreased $5.5 \%$ or $0.6 \%$ from $\$ 399.77$ to $\$ 377.94$. However, prior to inflation-adjustment, there was an increase of $10.6 \%$ and $1.1 \%$ from $\$ 341.69$ in 2009 to
$\$ 377.94$ in 2018. The average charges per visit, included multiple procedures in multiple regions; however, limited to epidural procedures only, showed the total costs after inflation-adjustment to decline $8 \%$ or $0.9 \%$ from $\$ 503.84$ in 2009 to $\$ 463.37$ in 2018. Prior to inflationadjustment, they showed increases of $7.6 \%$ and $0.8 \%$ from $\$ 430.63$ to $\$ 463.37$ from 2009 to 2018 .

Average charges per patient also declined both prior to adjustment of the inflation and after the adjustment of inflation. Prior to adjustment of the inflation, they declined $3.1 \%$ or $0.3 \%$ from $\$ 845.24$ to $\$ 819.27$, whereas after inflation-adjustment, they declined $17.2 \%$ and $2.1 \%$ from $\$ 988.93$ to $\$ 819.27$.

Finally, the total allowed charges by place of ser- 
Pain Physician: January/February 2021 24:1-15

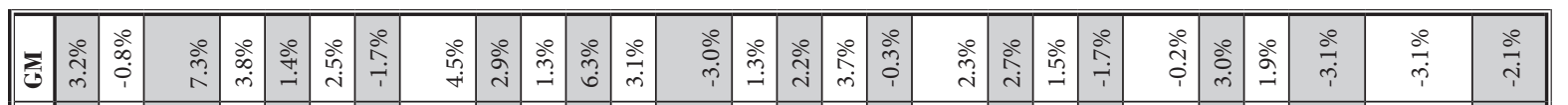

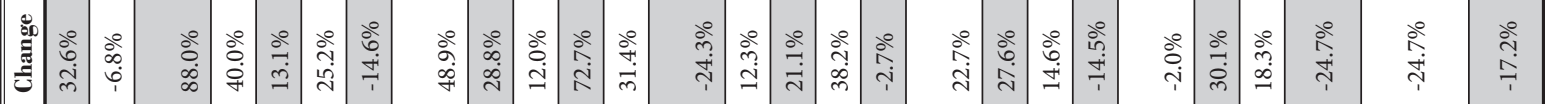

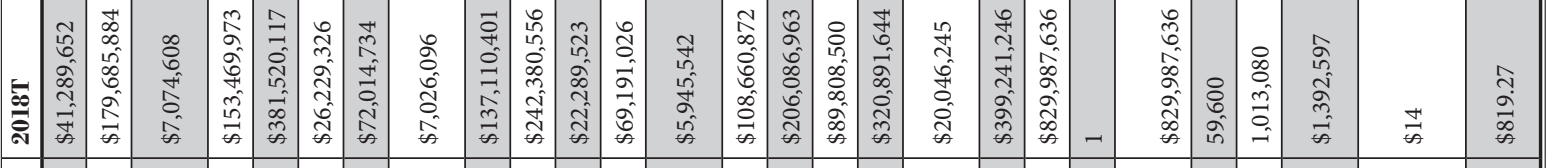

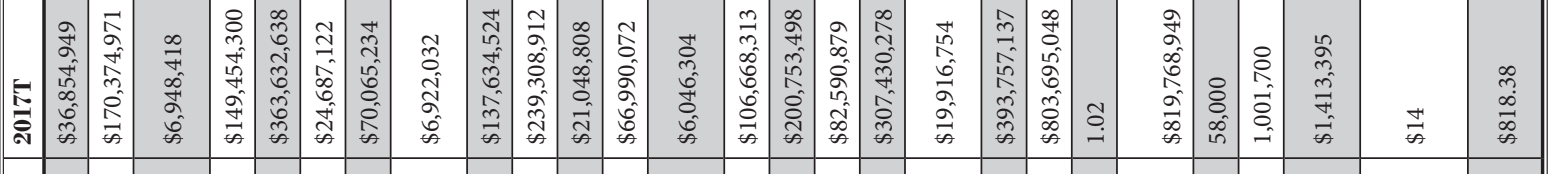

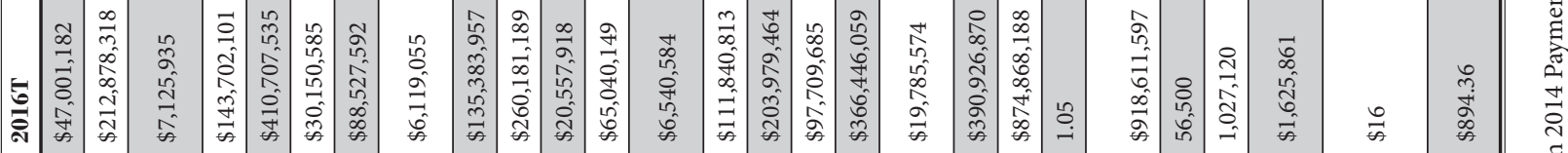

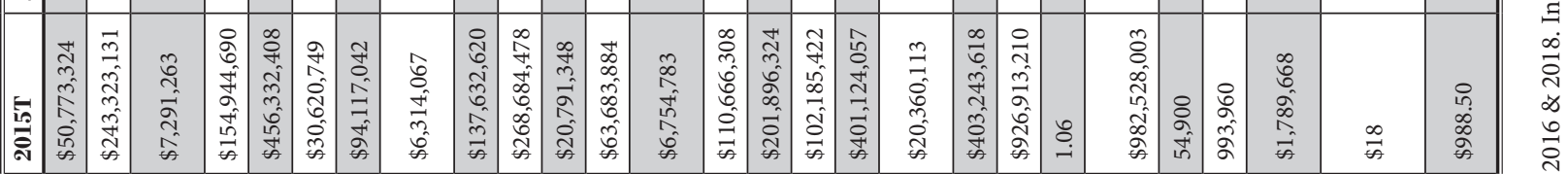

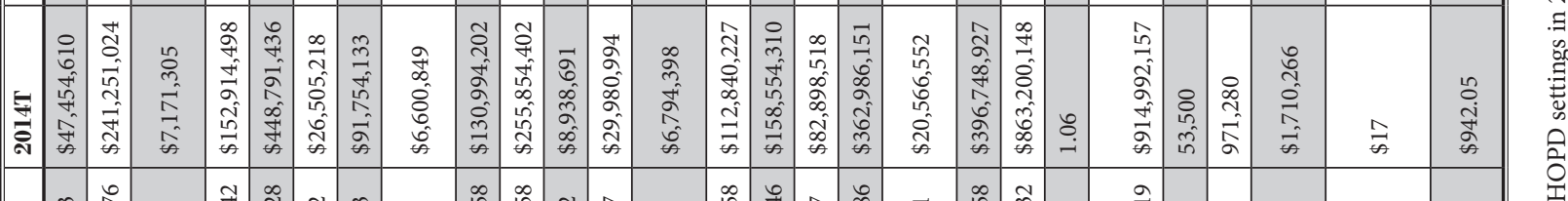

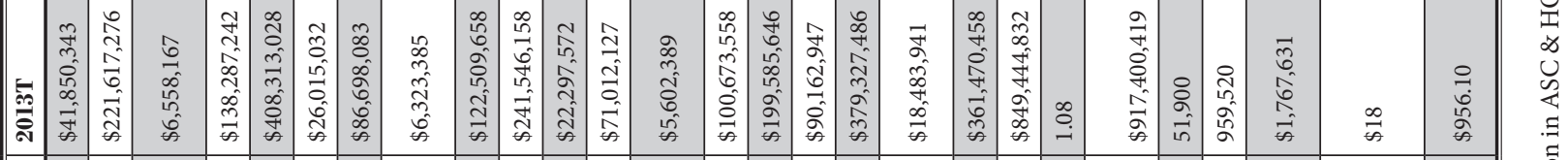

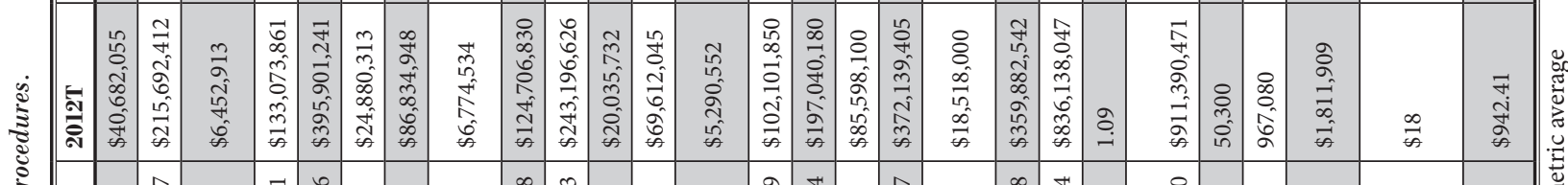

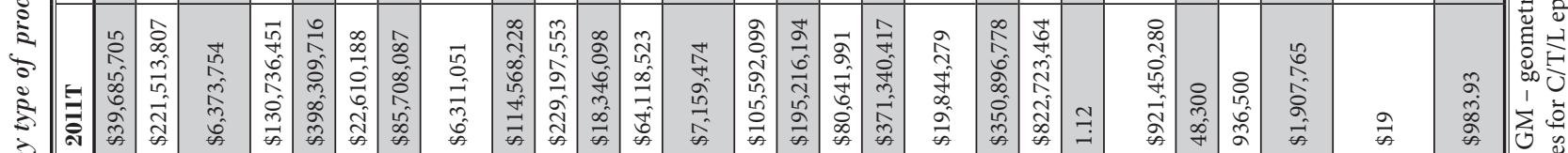

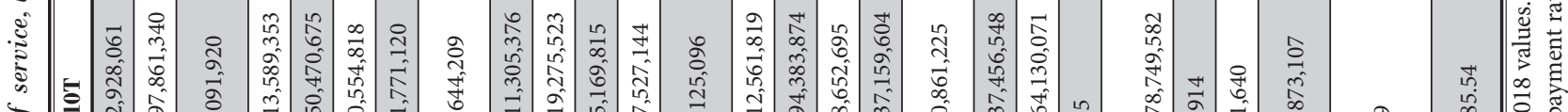

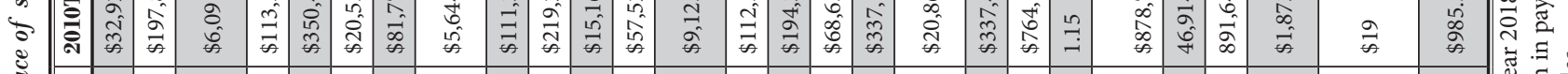

ङ

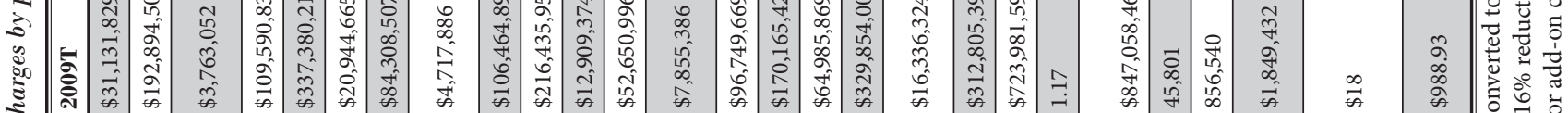

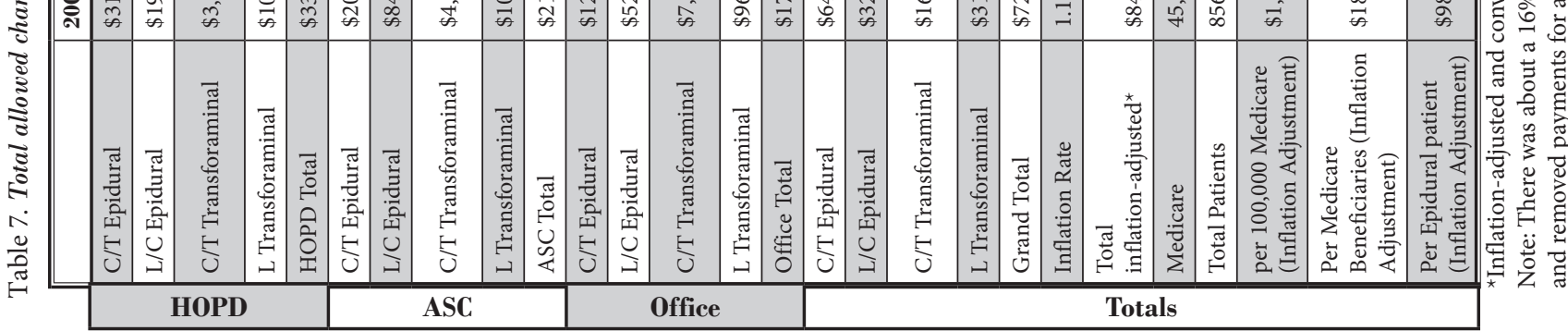


vice and type of procedures showed the total charges of \$723,981,594 in 2009 and \$829,987,636 in 2018 with a $14.6 \%$ and $1.5 \%$ increase. The overall allowed charges with inflation-adjusted rates showed a $2 \%$ decrease and $0.2 \%$ annually, decreasing from $\$ 847,058,465$ to $\$ 829,987,636$. Interestingly, the total allowed charges for lumbosacral caudal epidural procedures decreased from $\$ 329,854,009$ in 2009 to $\$ 320,891,064$ in 2018, with a decline of $2.7 \%$ and $0.3 \%$ annually. In contrast, for lumbosacral transforaminal epidural procedures, the increases were $27.6 \%$ with an annual increase of $2.7 \%$, changing from $\$ 312,805,391$ to $\$ 399,241,246$. In 2009, interlaminar and caudal epidural were the most commonly performed procedures; however, in 2018, the ratios almost reversed with the proportion of transforaminal epidurals decreasing from 1,395 per 100,000 Medicare population in 2009 to 1,304 compared to caudal and interlaminar epidural injections, which declined from 1,914 in 2009 to 1,277 in 2018 as shown in Tables 8, Fig. 3, and Appendix Tables 3 and 4.

Overall, in $2018,42 \%$ of the patients, or 752,980 received lumbar interlaminar and caudal epidural injections, whereas $43.2 \%$, or 773,200 received lumbosacral transforaminal epidural injections (Fig. 3 and Table 8).

Medicare is concerned with utilization patterns and the increasing expenditures of overall interventional techniques, even though they are showing a decline. Epidural procedures compared to overall interventional techniques or facet joint interventions are at a further decline. With the COVID-19 interface, these declines are going to be substantial. Consequently, CMS continues to update Local Coverage Determinations (LCDs) by calling for multijurisdictional Contractor Advisory Committee (CAC) assessment of present LCDs for epidural procedures, and performing enhanced audits, and investigations (9-12,47-56). The philosophy continues towards reducing the utilization and expenditures by not only reducing fraud and abuse, but also by enforcing the appropriate indications and medical necessity criteria. During the enactment of the ACA, or

Table 8. Proportion of patients and types of epidural procedures.

\begin{tabular}{|l|c|c||}
\hline \hline & $\begin{array}{c}\text { No. of } \\
\text { Patients }\end{array}$ & Percentage \\
\hline Cervical/Thoracic Transforaminal & 37,520 & $2.1 \%$ \\
\hline Cervical/Thoracic Epidural & 218,420 & $12.2 \%$ \\
\hline Lumbar/Caudal Epidural & 752,980 & $42.0 \%$ \\
\hline Lumbosacral Transforaminal & 773,200 & $43.2 \%$ \\
\hline
\end{tabular}

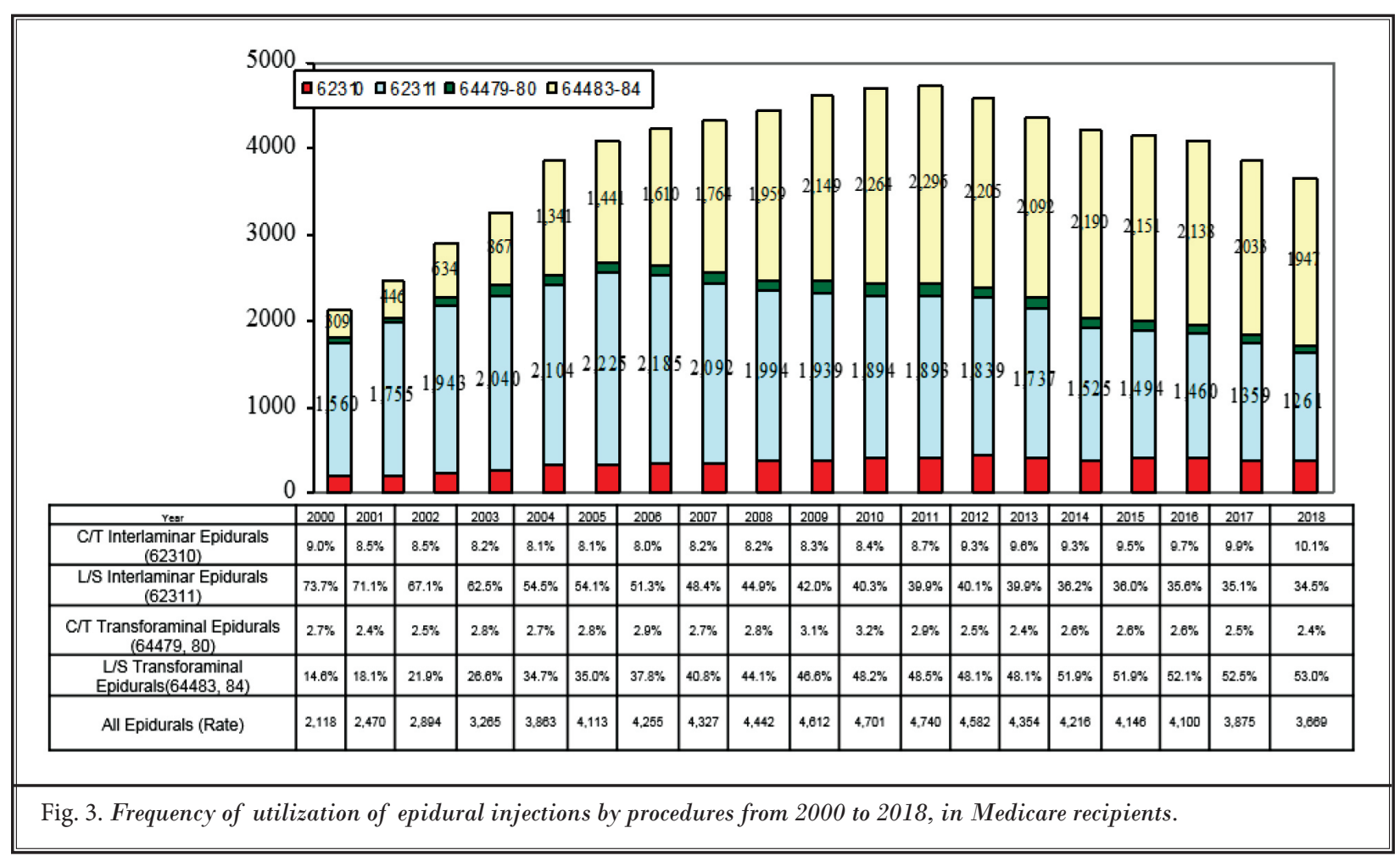


soon after, multiple MACs have enacted LCDs, which were essentially accepted verbatim by all carriers, except for CGS and First Coast Services, which essentially increased the frequency of epidural procedures from 4 to 6 in the therapeutic phase in all jurisdictions (49-54). CGS maintained 2 procedures in the diagnostic phase and 4 procedures in the therapeutic phase per rolling year (53). Thus, multijurisdictional CAC is being called to enact a national coverage policy and to provide uniform guidance (57).

This assessment showed the reversal of transforaminal epidural procedure utilization patterns and the increasing additional costs incurred for transforaminal epidural procedures (Table 7 and Appendix Tables 3 and 4). While multiple causes are considered for the decline in utilization, which is not yet the expected levels, due to overutilization, abuse, and fraud, as described above, which may be attributing to a lack of rapid decline and a growing Medicare population. In addition, the arguments continue in reference to indications and the medical necessity of epidural procedures and interventional techniques in general (7,58-80). Further, the disagreements and criticism continue in reference to the evidence, both positive and negative $(7,59-80)$.

\section{Conclusion}

The estimations of expenditures of epidural procedures in the Medicare FFS population from 2009 to 2018 showed declining net costs based on inflation-adjusted estimations. However, the costs increased without adjusting for inflation. Further, the utilization of these procedures was below the growth of the Medicare population, both in terms of net population and rate of population. This analysis of the FFS Medicare population from 2009 to 2018 demonstrated reduction in true utilization patterns of overall epidural injections, except for transforaminal epidural injections, which have increased, and with a change in the ratio of caudal and interlaminar epidural injections versus transforaminal epidural injections. The increases in expenditures also reflected these patterns. Overall, lumbar interlaminar epidural injections decreased from 1,914 per 100,000 Medicare population in 2009 to 1,277, with a $33.3 \%$ decrease, or an annual decrease of $4.4 \%$. However, lumbar transforaminal epidural injections showed a much lower decline from 1,395 in 2009 to 1,304 in 2018, with a decline of $6.5 \%$ and $0.7 \%$ annually. Overall, transforaminal epidural injections increased $27.6 \%$ and $2.7 \%$ per year, whereas for lumbosacral interlaminar epidural injections, they decreased overall $2.7 \%$ and $0.3 \%$ annu- ally while total expenditures increased $14.6 \%$ with an annual increase of $1.5 \%$. Inflation-adjusted expenses decreased $2 \%$ with an annual decline of $0.2 \%$.

\section{Acknowledgments}

The authors wish to thank Bert Fellows, MA, Director Emeritus of Psychological Services, Pain Management Centers of America, for manuscript review, and Tonie M. Hatton and Diane E. Neihoff, transcriptionists, for their assistance in preparation of this manuscript. We would like to thank the editorial board of Pain Physician for review and criticism in improving the manuscript.

\section{Author Contributions}

The study was designed by LM, VP, and JAH.

Data collection and analysis was performed by VP.

All authors contributed to the preparation of the manuscript, review, and approval of the content with final version.

Dr. Manchikanti is Co-Director, Pain Management Centers of America, Paducah, KY, Clinical Professor, Anesthesiology and Perioperative Medicine, University of Louisville, Louisville, KY, and Professor of Anesthesiology-Research, Department of Anesthesiology, School of Medicine, LSU Health Sciences Center, New Orleans and Shreveport, LA.

drlm@thepainmd.com

Vidyasagar Pampati is a Statistician, Pain Management Centers of America

sagar@thepainmd.com

Dr. Soin is Medical Director, Ohio Pain Clinic and Clinical Assistant Professor of Surgery at Wright State University, Dayton, $\mathrm{OH}$ Assistant Professor, Ohio University College of Medicine, Athens, $\mathrm{OH}$, and, Clinical Professor of Pain Management and Anesthesiology, University of Pikeville, Pikeville, KY

drsoin@gmail.com

Dr. Sanapati is Co-Director, Pain Management Centers of America, Evansville, IN, and Assistant Professor of Anesthesiology and Research, School of Medicine, LSU Health Sciences Center, New Orleans, LA. msanapati@gmail.com

Dr. Kaye is Professor, Pain Fellowship Director, Provost, Chief Academic Officer, Vice Chancellor of Academic Affairs, Departments of Anesthesiology and 
Pharmacology, Toxicology, and Neurosciences, LSU Health Sciences Center, Shreveport, Oshcher Shreveport Hospital and Pain Clinic Feist-Wieller Cancer Center, Shreveport, LA

akaye@|suhsc.edu, alankaye44@hotmail.com
Dr. Hirsch is Vice Chair and Service Line Chief of Neurointerventional Radiology, Chief of Neurointerventional Spine, Massachusetts General Hospital and Harvard Medical School, Boston, MA.

jahirsch@mgh.harvard.edu

Appendix tables are available at painphysicianjournal.com

\section{References}

1. Manchikanti L, Sanapati MR, Pampati V, Boswell MV, Kaye AD, Hirsch JA. Update on reversal and decline of growth of utilization of interventional techniques in managing chronic pain in the Medicare population from 2000 to 2018. Pain Physician 2019; 22:521-536.

2. Manchikanti L, Sanapati MR, Soin A, et al. An updated analysis of utilization of epidural procedures in managing chronic pain in the Medicare population from 2000 to 2018. Pain Physician 2020; 23:111-126.

3. Manchikanti L, Soin A, Mann DP, Bakshi S, Pampati V, Hirsch JA. Comparative analysis of utilization of epidural procedures in managing chronic pain in the Medicare population: Pre and post Affordable Care Act. Spine (Phila Pa 1976) 2019; 44:220-232.

4. Manchikanti L, Sanapati MR, Pampati $V$, et al. Update of utilization patterns of facet joint interventions in managing spinal pain from 2000 to 2018 in the US fee-for-service Medicare population. Pain Physician 2020; 23:E133-E149.

5. Manchikanti L, Manchikanti MV, Vanaparthy R, Kosanovic R, Pampati V. Utilization patterns of sacroiliac joint injections from 2000 to 2018 in feefor-service Medicare population. Pain Physician 2020; 23:439-450.

6. Manchikanti L, Kosanovic R, Pampati $\mathrm{V}$, Kaye AD. Declining utilization patterns of percutaneous adhesiolysis procedures in the United States feefor-service (FFS) Medicare population. Pain Physician 2021; 24:17-29.

7. Manchikanti L, Kaye A, Soin A, et al. Comprehensive evidence-based guidelines for facet joint interventions in the management of chronic spinal pain: American Society of Interventional Pain Physicians (ASIPP) guidelines. Pain Physician 2020; 23:S1-S127.

8. Hoyt D, Urits I, Orhurhu V, et al.
Current concepts in the management of vertebral compression fractures. Curr Pain Headache Rep 2020; 24:16.

9. Manchikanti L, Helm S 2nd, Benyamin RM, Hirsch JA. A critical analysis of Obamacare: Affordable care or insurance for many and coverage for few? Pain Physician 2017; 20:111-138.

10. Obama B. United States health care reform: Progress to date and next steps. JAMA 2016; 316:525-532.

11. Blumenthal D, Collins SR, Fowler EJ. The Affordable Care Act at 10 Years - Its coverage and access provisions. $N$ Engl J Med 2020; 382:963-969.

12. Peterson MA. The ACA a decade in: Resilience, impact, and vulnerabilities. J Health Polit Policy Law 2020; 45:595-608.

13. Keehan SP, Cuckler GA, Poisal JA, et al. National health expenditure projections, 2019-28: Expected rebound in prices drives rising spending growth. Health Aff (Millwood) 2020; 39:704-714.

14. Manchikanti L, Pampati V, Falco FJE, Hirsch JA. Growth of spinal interventional pain management techniques: Analysis of utilization trends and Medicare expenditures 2000 to 2008. Spine (Phila Pa 1976) 2013; 38:157-168.

15. Dieleman JL, Cao J, Chapin A, et al. US health care spending by payer and health condition, 1996-2016. JAMA 2020; 323:863-884.

16. Dieleman JL, Baral R, Birger $M$, et al. US spending on personal health care and public health, 1996-2013. JAMA 2016; 316:2627-2646.

17. U.S. Burden of Disease Collaborators. The state of US health, 1990-2010: Burden of diseases, injuries, and risk factors. JAMA 2013; 310:591-608.

18. Manchikanti L, Kaye AM, Knezevic NN, et al. Responsible, safe, and effective prescription of opioids for chronic non-cancer pain: American Society of Interventional Pain Physicians (ASIPP) guidelines. Pain Physician 2017; 20: $\mathrm{S}_{3}-\mathrm{S}_{92}$.

19. Navani A, Manchikanti L, Albers SL, et al. Responsible, safe, and effective use of biologics in the management of low back pain: American Society of Interventional Pain Physicians (ASIPP) guidelines. Pain Physician 2019; 22:S1-S74.

20. U.S. Department of Health and Human Services. Pain Management Best Practices Inter-Agency Task Force. Final Report on Pain Management Best Practices: Updates, Gaps, Inconsistencies, and Recommendations. May 9, 2019. www. hhs.gov/ash/advisory-committees/ pain/reports/index.html. Accessed 11/16/2020.

21. Manchikanti L, Singh V, Kaye AD, Hirsch JA. Lessons for better pain management in the future: Learning from the past. Pain Ther 2020; 9:373-391.

22. Manchikanti L, Vanaparthy R, Atluri S, Sachdeva H, Kaye AD, Hirsch JA. Concurrent public health emergencies in the US: COVID-19 and the opioid epidemic with hampered access to chronic pain management: A review. Pain Ther 2020; in press.

23. Gharibo C, Sharma A, Soin A, et al. Triaging interventional pain procedures during COVID-19 or related elective surgery restrictions: Evidence-informed guidance from the American Society of Interventional Pain Physicians (ASIPP). Pain Physician 2020; 23:S183-S2O4.

24. Shah $S$, Diwan $S$, Soin $A$, et al. Evidence-informed risk mitigation and stratification during COVID-19 for return to interventional pain practice: American Society of Interventional Pain Physicians (ASIPP) guidelines. Pain Physician 2020; 23:S161-S182. 
25. Kaye AD, Manchikanti L. COVID-19 Special Issue Editorial. Pain Physician 2020; 23:S159-S160.

26. Soin A, Vuppala S, Surfield G, et al. Ohio response to COVID-19 and its impact on interventional pain management practices. Pain Physician 2020; 23:S439-S448.

27. Wahezi SE, Duerte RA, Yerra $S$, et al. Telemedicine during COVID-19 and beyond: A practical guide and best practices multidisciplinary approach for the orthopedic and neurologic pain physical examination. Pain Physician 2020; 23:S205-S238.

28. Jha S, Shah S, Calderon MD, Soin A, Manchikanti L. The effect of COVID-19 on interventional pain management practices: A physician burnout survey. Pain Physician 2020; 23:S271-S282.

29. Gharaei H, Diwan S. COVID-19 Pandemic: Implications on interventional pain practice-A narrative review. Pain Physician 2020; 23:S $311-S_{318}$

30. Shah S, Diwan S, Kohan L, et al. The technological impact of COVID-19 on the future of education and health care delivery. Pain Physician 2020; 23: $\mathrm{S}_{3} 67-\mathrm{S}_{3} 8 \mathrm{O}$.

31. Prem A, Anand S, Chandrashekar A. Impact of COVID-19 on an academic pain fellowship program. Pain Physician 2020; 23:S433-S438.

32. American Medical Association. COVID-19 physician practice financial impact survey results. Accessed 11/3/2020. www.ama-assn.org/ practice-management/sustainability/ covid-19-physician-practice-financialimpact-survey-results

33. Slavitt A, Mostashari F. Covid-19 is battering independent physician practices. They need help now. STAT, May 28, 2020. Accessed 11/3/2020. www.statnews.com/2020/05/28/covid19-battering-independent-physicianpractices/

34. Manchikanti L, Falco FJE, Pampati V, Cash KA, Benyamin RM, Hirsch JA. Cost utility analysis of caudal epidural injections in the treatment of lumbar disc herniation, axial or discogenic low back pain, central spinal stenosis, and post lumbar surgery syndrome. Pain Physician 2013; 16:E129-E143.

35. Manchikanti L, Pampati V, Benyamin RM, Hirsch JA. Cost utility analysis of lumbar interlaminar epidural injections in the treatment of lumbar disc herniation, central spinal stenosis, and axial or discogenic low back pain.
Pain Physician 2017; 20:219-228.

36. Manchikanti L, Pampati V, Sanapati SP, Sanapati MR, Kaye AD, Hirsch JA. Evaluation of cost-utility of thoracic interlaminar epidural injections. Curr Pain Headache Rep 2020; 24:5.

37. Manchikanti L, Pampati V, Parr III A, et al. Cervical interlaminar epidural injections in the treatment of cervical disc herniation, post surgery syndrome, or discogenic pain: Cost utility analysis from randomized trials. Pain Physician 2019; 22:421-431.

38. Manchikanti L, Helm $S$ 2nd, Pampati V, Racz GB. Cost utility analysis of percutaneous adhesiolysis in managing pain of post-lumbar surgery syndrome and lumbar central spinal stenosis. Pain Pract 2015; 15:414-422.

39. Manchikanti L, Pampati V, Kaye AD, Hirsch JA. Therapeutic lumbar facet joint nerve blocks in the treatment of chronic low back pain: Cost utility analysis based on a randomized controlled trial. Korean J Pain 2018; 31:27-38.

40. Manchikanti L, Pampati V, Kaye AD, Hirsch JA. Cost utility analysis of cervical therapeutic medial branch blocks in managing chronic neck pain. Int J Med Sci 2017; 14:1307-1316.

41. Taylor RS, Ryan J, O'Donnell R, Eldabe $\mathrm{S}$, Kumar K, North RB. The costeffectiveness of spinal cord stimulation in the treatment of failed back surgery syndrome. Clin J Pain 2010; 26:463-469.

42. Tosteson AN, Skinner JS, Tosteson TDet al. The cost effectiveness of surgical versus nonoperative treatment for lumbar disc herniation over two years: evidence from the Spine Patient Outcomes Research Trial (SPORT). Spine (Phila Pa 1976) 2008; 33:2108-2115.

43. Tosteson AN, Lurie JD, Tosteson TD, et al; SPORT Investigators. Surgical treatment of spinal stenosis with and without degenerative spondylolisthesis: Cost-effectiveness after 2 years. Ann Intern Med 2008; 149:845-853.

44. Manchikanti L, Pampati V, Soin A, et al. Trends of expenditures and utilization of facet joint interventions in fee-forservice (FFS) Medicare population from 2009-2018. Pain Physician 2020; 23:S129-S147.

45. Vandenbroucke JP, von Elm E, Altman DG, et al; STROBE Initiative. Strengthening the Reporting of Observational Studies in Epidemiology (STROBE): Explanation and elaboration. Epidemiology 2007;
18:805-835

46. Centers for Medicare and Medicaid Services. Accessed 11/16/2020. www. cms.gov/

47. Manchikanti L, Helm II S, Singh V, Hirsch JA. Accountable interventional pain management: A collaboration among practitioners, patients, payers, and government. Pain Physician 2013; 16:E635-E670.

48. Manchikanti L, Singh V, Benyamin RM, Kaye AD, Pampati V, Hirsch JA. Reframing Medicare physician payment policy for 2019: A look at proposed policy. Pain Physician 2018; 21:415-432.

49. Noridian Healthcare Solutions, LLC. Local Coverage Determination (LCD): Lumbar Epidural Injections (L34982). Revision Effective Date: 10/1/2019.

50. First Coast Service Options, Inc. Epidural (L33906). Revision Effective Date: 10/01/2019.

51. Wisconsin Physician Services Insurance Corporation. Local Coverage Determination (LCD). Lumbar Epidural Injections (L36521). Effective Date: 11/1/2019.

52. Palmetto GBA. Local Coverage Determination (LCD): Lumbar Epidural Steroid Injections (L35148). Revision Effective Date: 10/24/2019.

53. CGS Administrators, LLC. Local Coverage Determination (LCD). Lumbar Epidural Steroid Injections (ESI) (L34807). Revision Effective Date: 12/26/2019.

54. National Government Services, Inc. Local Coverage Determination (LCD): Lumbar Epidural Injections (L35937). Revision Effective Date: 10/17/2019.

55. Manchikanti L, Falco FJE, Benyamin RM, Gharibo CG, Candido KD, Hirsch JA. Epidural steroid injections safety recommendations by the MultiSociety Pain Workgroup (MPW): More regulations without evidence or clarification. Pain Physician 2014; 17:E575-E588.

56. Nguyen TH, Milburn JM, Duszak R, Savoie J, Horńy M, Hirsch JA. Medicare for all: considerations for neuroradiologists. AJNR Am J Neuroradiol 2020; 41:772-776.

57. Multijurisdictional CAC Meeting for Epidural Injections, February 2021.

58. Chou R, Hashimoto R, Friedly JL, et al. Epidural corticosteroid injections for radiculopathy and spinal stenosis: A systematic review and meta-analysis. Ann Intern Med 2015; 163:373-381. 
59. Oliveira CB, Maher CG, Ferreira ML, et al. Epidural corticosteroid injections for lumbosacral radicular pain. Cochrane Database Syst Rev 2020; 4:CDo13577.

6o. Manchikanti L, Knezevic NN, Boswell MV, Kaye AD, Hirsch JA. Epidural injections for lumbar radiculopathy and spinal stenosis: A comparative systematic review and meta-analysis. Pain Physician 2016; 19:E365-E410.

61. Lee JH, Kim DH, Kim DH, et al. Comparison of clinical efficacy of epidural injection with or without steroid in lumbosacral disc herniation: A systematic review and meta-analysis. Pain Physician 2018; 21:449-468.

62. Manchikanti L, Singh V, Pampati V, Falco FJE, Hirsch JA. Comparison of the efficacy of caudal, interlaminar, and transforaminal epidural injections in managing lumbar disc herniation: Is one method superior to the other? Korean J Pain 2015; 28:11-21.

63. Knezevic N, Manchikanti L, Urits I, et al. Lack of superiority of epidural injections with lidocaine with steroids compared to without steroids in spinal pain: A systematic review and meta-analysis. Pain Physician 2020; 23:S239-S270.

64. Manchikanti L, Knezevic NN, Parr A, Kaye AD, Sanapati M, Hirsch JA. Does epidural bupivacaine with or without steroids provide long-term relief? A systematic review and meta-analysis. Curr Pain Headache Rep 2020; 24:26.

65. Manchikanti L, Kosanovic R, Vanaparthy $R$, et al. Steroid distancing in interventional pain management during COVID-19 and beyond: Safe, effective and practical approach. Pain Physician 2020; 23:S319-S352.

66. Manchikanti L, Nampiaparampil DE, Candido KD, et al. Do cervical epidural injections provide long-term relief in neck and upper extremity pain? A systematic review. Pain Physician 2015; 18:39-60.

67. Manchikanti L, Nampiaparampil DE,
Manchikanti KN, et al. Comparison of the efficacy of saline, local anesthetics, and steroids in epidural and facet joint injections for the management of spinal pain: A systematic review of randomized controlled trials. Surg Neurol Int 2015; 6:S194-S235.

68. Manchikanti L, Benyamin RM, Falco FJ, Kaye AD, Hirsch JA. Do epidural injections provide short- and longterm relief for lumbar disc herniation? A systematic review. Clin Orthop Relat Res 2015; 473:1940-1956.

69. Lee $J H$, Shin $K H$, Bahk SJ, et al. Comparison of clinical efficacy of transforaminal and caudal epidural steroid injection in lumbar and lumbosacral disc herniation: A systematic review and meta-analysis. Spine ] 2018; 18:2343-2353.

70. Lee JH, Shin KS, Park SJ, et al. Comparison of clinical efficacy between transforaminal and interlaminar epidural injections in lumbosacral disc herniation: A systematic review and meta-analysis. Pain Physician 2018; 21:433-448.

71. Shanthanna H, Busse J, Wang L, et al. Addition of corticosteroids to local anaesthetics for chronic non-cancer pain injections: A systematic review and meta-analysis of randomised controlled trials. Br J Anaesth 2020; 125:779-801.।

72. Zhao W, Wang Y, Wu J, et al. Long-term outcomes of epidurals with lidocaine with or without steroids for lumbar disc herniation and spinal stenosis: A meta-analysis. Pain Physician 2020; 23:365-374.

73. Mesregah MK, Feng W, Huang WH, et al. Clinical effectiveness of interlaminar epidural injections of local anesthetic with or without steroids for managing chronic neck pain: A systematic review and meta-analysis. Pain Physician 2020; 23:335-348.

74. Manchikanti L, Knezevic NN, Sanapati SP, Sanapati MR, Kaye AD, Hirsch JA. Is percutaneous adhesiolysis effective in managing chronic low back and lower extremity pain in post-surgery syndrome: A systematic review and meta-analysis. Curr Pain Headache Rep 2020; 24:30.

75. Manchikanti L, Knezevic NN, Sanapati MR, Boswell MV, Kaye AD, Hirsch JA. Effectiveness of percutaneous adhesiolysis in managing chronic central lumbar spinal stenosis: A systematic review and meta-analysis. Pain Physician 2019; 22:E523-E550.

76. Manchikanti L, Soin A, Boswell MV, Kaye AD, Sanapati M, Hirsch JA. Effectiveness of percutaneous adhesiolysis in post lumbar surgery syndrome: A systematic analysis of findings of systematic reviews. Pain Physician 2019; 22:307-322.

77. Sanapati J, Manchikanti L, Atluri S, et al. Do regenerative medicine therapies provide long-term relief in chronic low back pain: A systematic review and metaanalysis. Pain Physician 2018; 21:515-540.

78. Chou R, Atlas SJ, Loeser JD, Rosenquist RW, Stanos SP. Guideline warfare over interventional therapies for low back pain: Can we raise the level of discourse? J Pain 2011; 12:833-839.

79. Manchikanti L, Benyamin RM, Falco FJE, Caraway DL, Datta S, Hirsch JA. Guidelines warfare over interventional techniques: Is there a lack of discourse or straw man? Pain Physician 2012; 15:E1-E26.

80. Boswell MV, Manchikanti L. Appropriate design and methodologic quality assessment, clinically relevant outcomes are essential to determine the role of epidural corticosteroid injections. Commentary RE: Chou R, Hashimoto R, Friedly JL, Fu R, Bougatsos C, Dana T, Sullivan SD, Jarvik J. Epidural corticosteroid injections for radiculopathy and spinal stenosis: $A$ systematic review and meta-analysis. Ann Intern Med 2015; 163:373-381. Evid Based Med 2016; 21:89. 



\begin{tabular}{|c|c|c|c|c|c|c|c|c|c|c|c|c|c|c|c|c|c|c|c|c|c|c|c|c|c|}
\hline 定 & $\mid \begin{array}{l}\mid \\
\text { oे } \\
i \\
i\end{array}$ & $\begin{array}{l}\text { oें } \\
\text { ஸे }\end{array}$ & $\begin{array}{c}\stackrel{0}{0} \\
\stackrel{-}{I}\end{array}$ & $\begin{array}{l}0 \\
\stackrel{\dot{m}}{0} \\
\end{array}$ & ঙें & 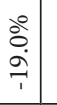 & $\stackrel{\text { هे }}{\text {. }}$ & ڤे & $\begin{array}{c}0 \\
\infty \\
\infty \\
\infty \\
i \\
1\end{array}$ & \begin{tabular}{c|}
0 \\
$\dot{\partial}$ \\
$i$ \\
$i$
\end{tabular} & $\begin{array}{l}\stackrel{\circ}{0} \\
\overrightarrow{3} \\
\rightarrow 1\end{array}$ & $\stackrel{\circ}{\stackrel{े}{+}}$ & $\begin{array}{l}\text { aे } \\
\hat{i} \\
1\end{array}$ & \begin{tabular}{l|l}
$\stackrel{0}{\circ}$ \\
\multirow{i}{*}{}
\end{tabular} & $\begin{array}{l}0 \\
\dot{b} \\
i \\
i\end{array}$ & $\stackrel{\text { aे }}{\rightarrow}$ & $\mid \begin{array}{c}\stackrel{0}{0} \\
\stackrel{0}{+} \\
+\end{array}$ & 今े & 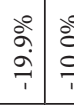 & 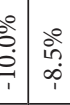 & 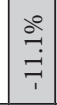 & 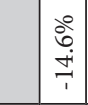 & $\begin{array}{c}0 \\
\grave{i} \\
0 \\
0 \\
i\end{array}$ & 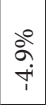 & 总 \\
\hline 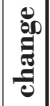 & ๙ें & $\underset{\infty}{\infty}$ & \begin{tabular}{|c|}
$\stackrel{0}{0}$ \\
$\infty$ \\
$\infty$ \\
$\sim$
\end{tabular} & $\begin{array}{l}\stackrel{0}{0} \\
\infty \\
\infty\end{array}$ & ڤे̀ & $\begin{array}{l}0 \\
0 \\
0 \\
0 \\
1 \\
1\end{array}$ & $\stackrel{\circ}{\circ}$ & 定 & $\begin{array}{l}20 \\
2 \\
1 \\
1\end{array}$ & 今े & 斑 & ๙ें & 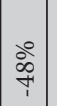 & 吕 & $\frac{\partial}{\hat{T}}$ & 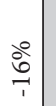 & 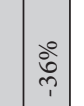 & 今े & \begin{tabular}{l|ll}
0 \\
\\
0
\end{tabular} & $\begin{array}{c}0 \\
\text { con } \\
1\end{array}$ & $\mid \begin{array}{l}0 \\
0 \\
i \\
i\end{array}$ & 용 & 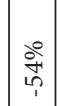 & $\begin{array}{l}80 \\
0 \\
\text { Dे }\end{array}$ & \\
\hline
\end{tabular}

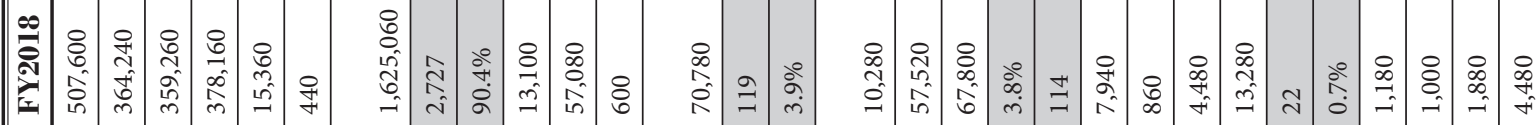

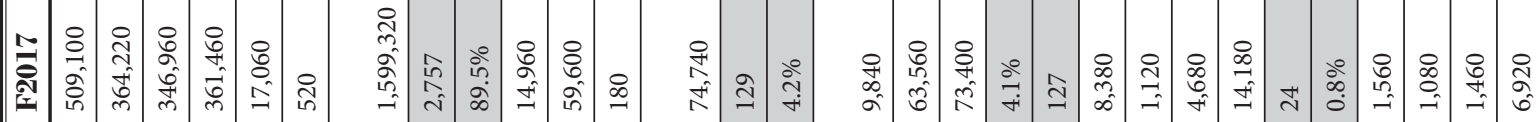

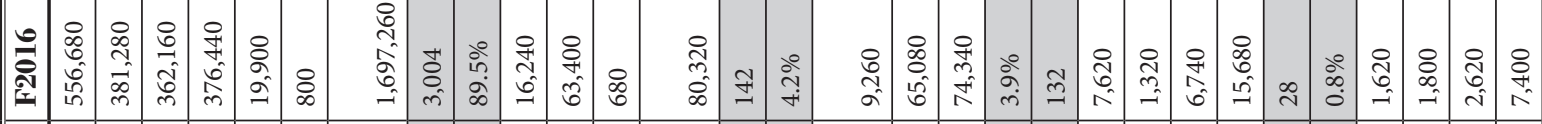

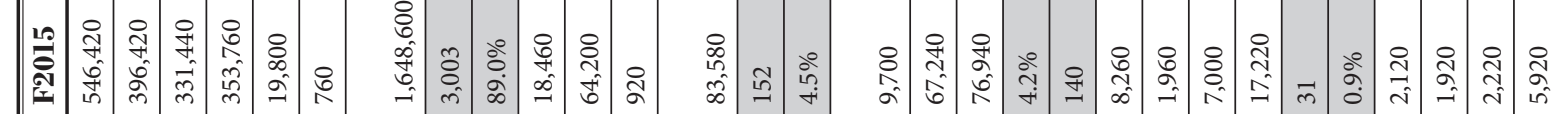
8

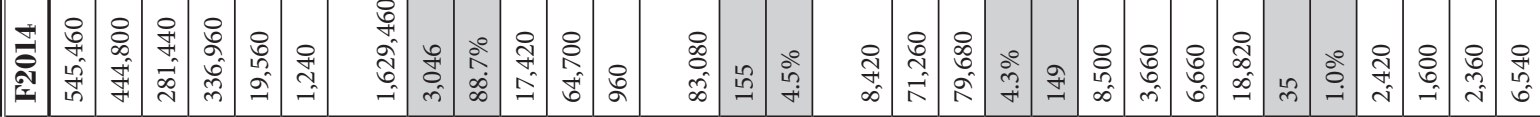
(n) క్

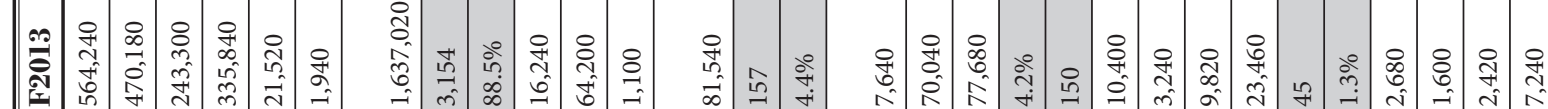
趾 के

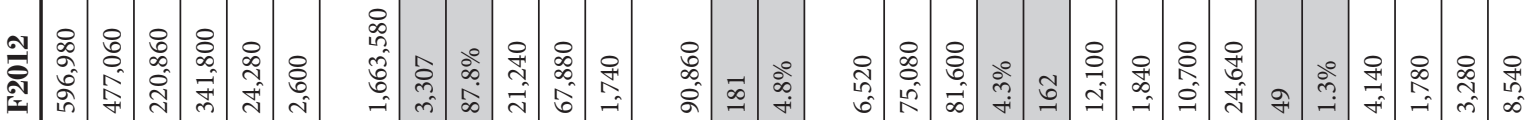
3

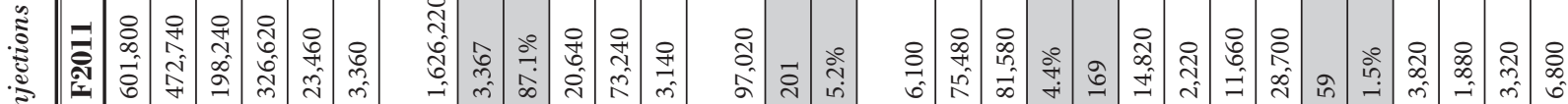
$\vec{\Xi}$

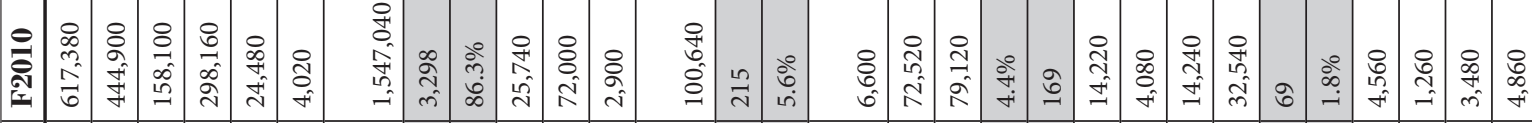
$\stackrel{2}{=}$

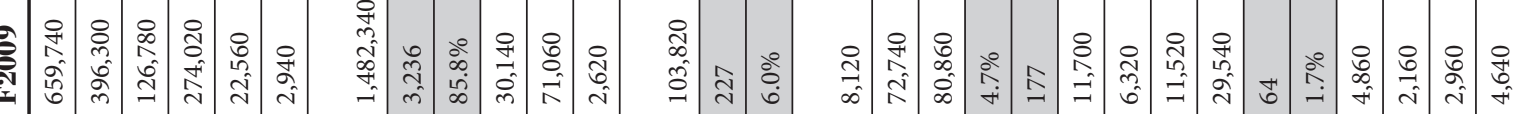

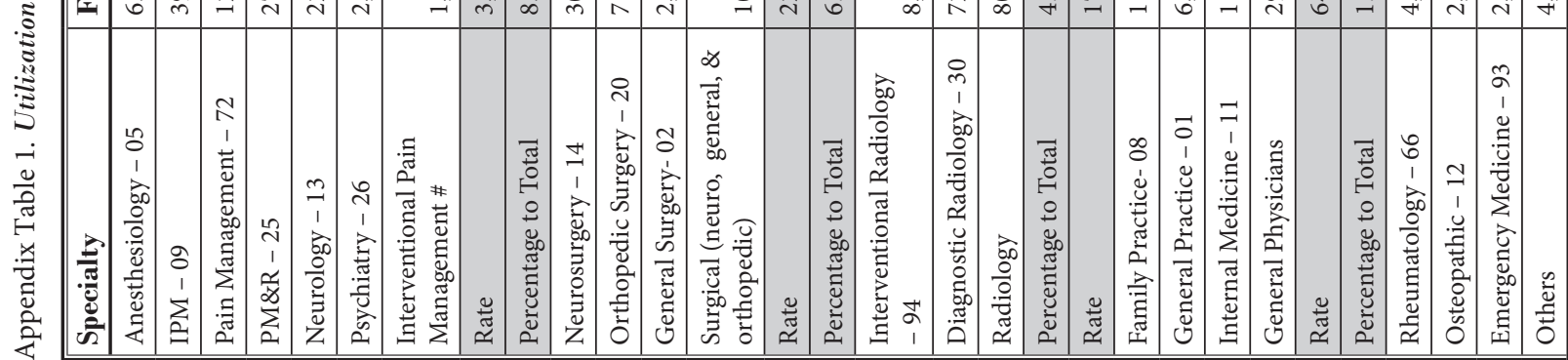




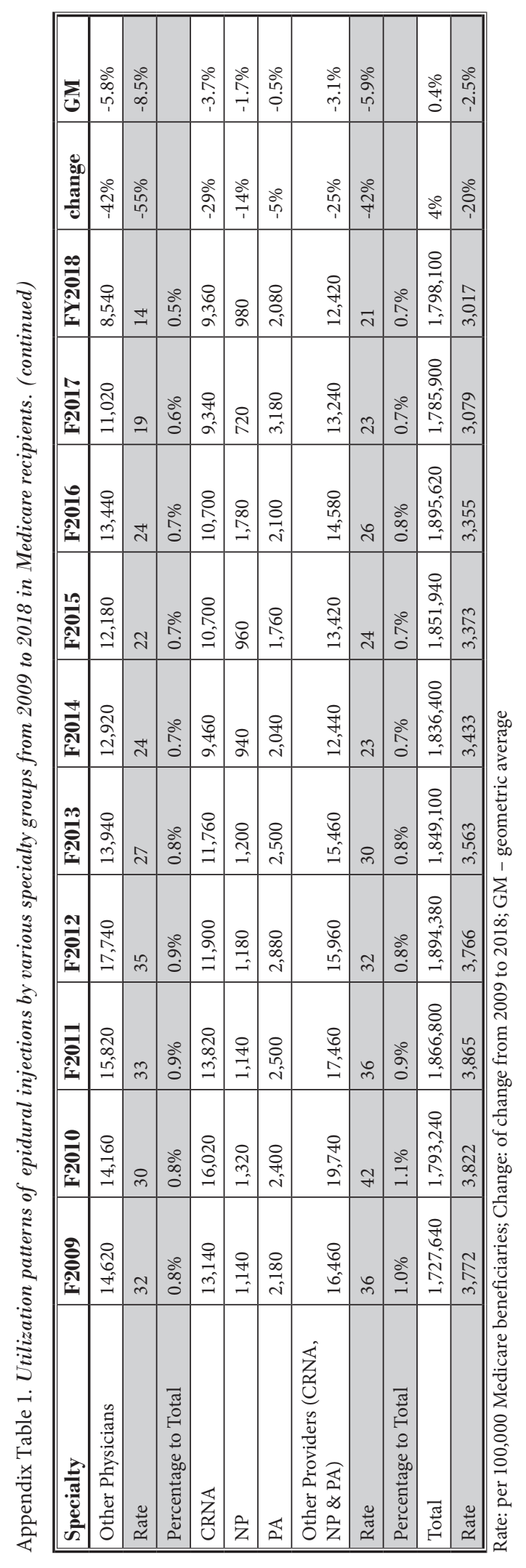


Declining Expenditures for Epidural Procedures in the Medicare Population

Appendix Table 2. Utilizations of epidural procedures (rate per 100,000) in the Medicare population from 2009 to 2018 (2016 Medicare carrier).

\begin{tabular}{|c|c|c|c|c|c|c|c|c|c|c|c|c|}
\hline State name & F2009 & F2010 & F2011 & F2012 & F2013 & F2014 & F2015 & F2016 & F2017 & F2018 & Change & $\overline{\text { GM }}$ \\
\hline \multicolumn{13}{|l|}{ Cahaba } \\
\hline Alabama & 5,723 & 5,496 & 5,927 & 5,943 & 5,775 & 5,715 & 5,622 & 5,814 & 4,705 & 4,685 & $-18.1 \%$ & $-2.2 \%$ \\
\hline Georgia & 5,270 & 4,988 & 5,176 & 5,191 & 4,437 & 4,253 & 4,132 & 3,922 & 3,766 & 3,589 & $-31.9 \%$ & $-4.2 \%$ \\
\hline Tennessee & 3,840 & 3,577 & 3,516 & 3,401 & 2,893 & 2,876 & 2,825 & 2,897 & 2,598 & 2,552 & $-33.5 \%$ & $-4.4 \%$ \\
\hline Cahaba Total & 4,910 & 4,649 & 4,819 & 4,791 & 4,271 & 4,175 & 4,084 & 4,074 & 3,622 & 3,527 & $-28.2 \%$ & $-3.6 \%$ \\
\hline PCPY & & $-5.3 \%$ & $3.6 \%$ & $-0.6 \%$ & $-10.9 \%$ & $-2.3 \%$ & $-2.2 \%$ & $-0.2 \%$ & $-11.1 \%$ & $-2.6 \%$ & & \\
\hline \multicolumn{13}{|l|}{ CGS } \\
\hline Kentucky & 4,385 & 5,108 & 5,062 & 5,052 & 4,690 & 4,378 & 4,050 & 4,165 & 3,880 & 3,892 & $-11.2 \%$ & $-1.3 \%$ \\
\hline Ohio & 3,671 & 3,524 & 3,441 & 3,470 & 3,296 & 3,146 & 2,929 & 3,195 & 2,683 & 2,522 & $-31.3 \%$ & $-4.1 \%$ \\
\hline Total & 3,874 & 3,977 & 3,906 & 3,924 & 3,698 & 3,501 & 3,251 & 3,473 & 3,024 & 2,912 & $-24.8 \%$ & $-3.1 \%$ \\
\hline PCPY & & $2.6 \%$ & $-1.8 \%$ & $0.5 \%$ & $-5.8 \%$ & $-5.3 \%$ & $-7.1 \%$ & $6.8 \%$ & $-12.9 \%$ & $-3.7 \%$ & & \\
\hline \multicolumn{13}{|l|}{ First Coast } \\
\hline Florida & 4,433 & 4,445 & 4,198 & 4,111 & 3,760 & 3,725 & 3,587 & 3,622 & 3,197 & 3,115 & $-29.7 \%$ & $-3.8 \%$ \\
\hline PCPY & & $0.3 \%$ & $-5.6 \%$ & $-2.1 \%$ & $-8.5 \%$ & $-0.9 \%$ & $-3.7 \%$ & $1.0 \%$ & $-11.7 \%$ & $-2.5 \%$ & & \\
\hline \multicolumn{13}{|l|}{ NGS } \\
\hline Connecticut & 2,928 & 2,897 & 3,339 & 2,953 & 2,841 & 2,756 & 2,569 & 2,614 & 2,435 & 2,189 & $-25.2 \%$ & $-3.2 \%$ \\
\hline Illinois & 4,262 & 4,406 & 4,715 & 4,605 & 4,600 & 4,159 & 4,040 & 4,083 & 3,774 & 3,585 & $-15.9 \%$ & $-1.9 \%$ \\
\hline Maine & 3,790 & 3,383 & 3,369 & 3,313 & 3,014 & 3,390 & 2,866 & 2,683 & 2,415 & 1,956 & $-48.4 \%$ & $-7.1 \%$ \\
\hline Massachusetts & 3,002 & 3,084 & 3,363 & 3,515 & 3,557 & 3,315 & 3,358 & 3,230 & 3,059 & 2,934 & $-2.3 \%$ & $-0.3 \%$ \\
\hline Minnesota & 2,441 & 2,222 & 2,165 & 2,179 & 2,241 & 1,933 & 1,877 & 1,609 & 1,398 & 1,283 & $-47.5 \%$ & $-6.9 \%$ \\
\hline New Hampshire & 3,423 & 3,762 & 4,166 & 3,664 & 3,617 & 3,733 & 3,484 & 3,644 & 3,239 & 3,260 & $-4.8 \%$ & $-0.5 \%$ \\
\hline New York & 2,499 & 2,346 & 2,346 & 2,375 & 2,528 & 2,400 & 2,447 & 2,339 & 2,116 & 2,107 & $-15.7 \%$ & $-1.9 \%$ \\
\hline Rhode Island & 2,486 & 2,995 & 2,521 & 1,984 & 1,727 & 1,979 & 2,274 & 1,889 & 1,550 & 1,877 & $-24.5 \%$ & $-3.1 \%$ \\
\hline Vermont & 2,353 & 2,439 & 2,390 & 2,300 & 2,852 & 2,474 & 2,694 & 2,405 & 1,968 & 2,299 & $-2.3 \%$ & $-0.3 \%$ \\
\hline Wisconsin & 3,411 & 3,528 & 3,401 & 3,218 & 3,213 & 2,827 & 2,643 & 2,754 & 2,590 & 2,507 & $-26.5 \%$ & $-3.4 \%$ \\
\hline NGS Total & 3,096 & 3,083 & 3,189 & 3,126 & 3,171 & 2,950 & 2,895 & 2,833 & 2,596 & 2,501 & $-19.2 \%$ & $-2.3 \%$ \\
\hline PCPY & & $-0.4 \%$ & $3.4 \%$ & $-2.0 \%$ & $1.4 \%$ & $-7.0 \%$ & $-1.8 \%$ & $-2.2 \%$ & $-8.4 \%$ & $-3.6 \%$ & & \\
\hline \multicolumn{13}{|l|}{ Noridain } \\
\hline Alaska & 3,189 & 3,532 & 3,224 & 3,348 & 3,359 & 3,958 & 4,064 & 3,744 & 3,243 & 3,907 & $22.5 \%$ & $2.3 \%$ \\
\hline Arizona & 3,593 & 4,089 & 4,048 & 4,487 & 4,410 & 4,296 & 4,288 & 4,103 & 3,959 & 3,984 & $10.9 \%$ & $1.2 \%$ \\
\hline California & 2,620 & 2,696 & 2,770 & 2,801 & 2,705 & 2,619 & 2,591 & 2,503 & 2,182 & 2,161 & $-17.5 \%$ & $-2.1 \%$ \\
\hline Idaho & 3,415 & 3,081 & 3,788 & 4,175 & 3,641 & 3,614 & 3,571 & 3,645 & 3,055 & 2,885 & $-15.5 \%$ & $-1.9 \%$ \\
\hline Montana & 3,596 & 3,493 & 3,754 & 3,509 & 3,426 & 3,382 & 3,336 & 3,298 & 2,821 & 3,031 & $-15.7 \%$ & $-1.9 \%$ \\
\hline Nevada & 3,766 & 3,124 & 3,669 & 3,844 & 3,703 & 3,466 & 3,493 & 3,285 & 2,811 & 2,755 & $-26.8 \%$ & $-3.4 \%$ \\
\hline North Dakota & 3,611 & 4,318 & 4,451 & 4,096 & 4,296 & 4,420 & 3,678 & 3,785 & 3,078 & 3,007 & $-16.7 \%$ & $-2.0 \%$ \\
\hline Oregon & 1,916 & 1,922 & 1,970 & 2,175 & 2,211 & 1,701 & 1,684 & 1,535 & 1,295 & 1,393 & $-27.3 \%$ & $-3.5 \%$ \\
\hline South Dakota & 4,031 & 3,456 & 4,142 & 3,913 & 3,425 & 3,787 & 4,102 & 3,715 & 3,253 & 3,463 & $-14.1 \%$ & $-1.7 \%$ \\
\hline Utah & 4,528 & 4,226 & 4,562 & 4,956 & 5,152 & 4,880 & 5,285 & 4,465 & 4,426 & 4,550 & $0.5 \%$ & $0.1 \%$ \\
\hline Washington & 2,844 & 3,011 & 2,635 & 2,887 & 2,776 & 2,568 & 2,351 & 2,259 & 2,038 & 1,943 & $-31.7 \%$ & $-4.1 \%$ \\
\hline Wyoming & 3,656 & 4,271 & 4,345 & 4,710 & 4,284 & 4,258 & 3,629 & 4,902 & 3,904 & 4,239 & $15.9 \%$ & $1.7 \%$ \\
\hline Noridian Total & 2,895 & 2,971 & 3,036 & 3,164 & 3,076 & 2,948 & 2,910 & 2,788 & 2,486 & 2,488 & $-14.1 \%$ & $-1.7 \%$ \\
\hline PCPY & & $2.6 \%$ & $2.2 \%$ & $4.2 \%$ & $-2.8 \%$ & $-4.2 \%$ & $-1.3 \%$ & $-4.2 \%$ & $-10.8 \%$ & $0.1 \%$ & & \\
\hline
\end{tabular}


Pain Physician: January/February 2021 24:1-15

Appendix Table 2. Utilizations of epidural procedures (rate per 100,000) in the Medicare population from 2009 to 2018 (2016 Medicare carrier). (continued)

\begin{tabular}{|c|c|c|c|c|c|c|c|c|c|c|c|c|}
\hline State name & F2009 & F2010 & F2011 & F2012 & F2013 & F2014 & F2015 & F2016 & F2017 & F2018 & Change & $\overline{\text { GM }}$ \\
\hline \multicolumn{13}{|l|}{ Novitas } \\
\hline Arkansas & 3,855 & 4,117 & 4,082 & 4,153 & 4,055 & 4,028 & 4,169 & 4,474 & 4,343 & 4,617 & $19.8 \%$ & $2.0 \%$ \\
\hline Colorado & 3,658 & 3,966 & 4,058 & 3,935 & 3,686 & 3,634 & 3,251 & 3,303 & 2,697 & 2,669 & $-27.0 \%$ & $-3.4 \%$ \\
\hline Delaware & 4,177 & 4,769 & 5,062 & 4,768 & 5,212 & 5,189 & 5,183 & 5,440 & 4,515 & 4,704 & $12.6 \%$ & $1.3 \%$ \\
\hline $\begin{array}{l}\text { District of } \\
\text { Columbia }\end{array}$ & 1,904 & 2,867 & 2,708 & 3,766 & 4,660 & 4,593 & 4,254 & 3,483 & 2,548 & 3,047 & $60.1 \%$ & $5.4 \%$ \\
\hline Louisiana & 4,502 & 4,235 & 4,718 & 4,445 & 4,133 & 3,911 & 4,244 & 3,921 & 3,685 & 3,350 & $-25.6 \%$ & $-3.2 \%$ \\
\hline Maryland & 3,863 & 4,141 & 4,515 & 4,578 & 4,459 & 4,377 & 4,906 & 4,552 & 4,026 & 4,353 & $12.7 \%$ & $1.3 \%$ \\
\hline Mississippi & 5,385 & 6,014 & 5,989 & 5,809 & 5,184 & 5,429 & 5,727 & 5,332 & 4,762 & 4,812 & $-10.6 \%$ & $-1.2 \%$ \\
\hline New Jersey & 3,387 & 3,271 & 3,417 & 3,393 & 3,229 & 3,511 & 3,325 & 3,323 & 3,025 & 3,044 & $-10.1 \%$ & $-1.2 \%$ \\
\hline New Mexico & 3,081 & 2,744 & 2,859 & 2,933 & 2,539 & 2,390 & 2,279 & 2,576 & 2,167 & 2,079 & $-32.5 \%$ & $-4.3 \%$ \\
\hline Oklahoma & 4,846 & 5,057 & 5,518 & 5,627 & 5,607 & 5,729 & 5,786 & 5,467 & 5,267 & 5,445 & $12.3 \%$ & $1.3 \%$ \\
\hline Pennsylvania & 2,905 & 3,132 & 3,180 & 3,153 & 3,224 & 3,157 & 3,170 & 3,093 & 2,899 & 2,909 & $0.1 \%$ & $0.0 \%$ \\
\hline Texas & 4,664 & 4,609 & 4,795 & 4,281 & 4,019 & 3,874 & 3,844 & 3,720 & 3,282 & 2,969 & $-36.3 \%$ & $-4.9 \%$ \\
\hline Novitas Total & 3,941 & 4,042 & 4,217 & 4,054 & 3,904 & 3,870 & 3,896 & 3,795 & 3,418 & 3,358 & $-14.8 \%$ & $-1.8 \%$ \\
\hline PCPY & & $2.6 \%$ & $4.3 \%$ & $-3.9 \%$ & $-3.7 \%$ & $-0.9 \%$ & $0.7 \%$ & $-2.6 \%$ & $-10.0 \%$ & $-1.7 \%$ & & \\
\hline \multicolumn{13}{|l|}{ Palmetto GBA } \\
\hline North Carolina & 4,638 & 4,489 & 5,001 & 5,098 & 4,733 & 4,188 & 4,030 & 4,288 & 3,736 & 3,659 & $-21.1 \%$ & $-2.6 \%$ \\
\hline South Carolina & 6,102 & 6,313 & 6,525 & 6,607 & 6,455 & 5,907 & 6,185 & 6,065 & 5,487 & 5,419 & $-11.2 \%$ & $-1.3 \%$ \\
\hline Virginia & 4,163 & 4,014 & 3,991 & 4,007 & 4,137 & 4,270 & 4,163 & 4,311 & 4,042 & 3,932 & $-5.5 \%$ & $-0.6 \%$ \\
\hline West Virginia & 2,386 & 2,268 & 2,362 & 2,515 & 2,430 & 2,141 & 2,270 & 2,265 & 1,730 & 1,857 & $-22.2 \%$ & $-2.7 \%$ \\
\hline Palmetto Total & 4,562 & 4,495 & 4,748 & 4,825 & 4,696 & 4,379 & 4,360 & 4,480 & 4,019 & 3,956 & $-13.3 \%$ & $-1.6 \%$ \\
\hline PCPY & & $-1.5 \%$ & $5.6 \%$ & $1.6 \%$ & $-2.7 \%$ & $-6.8 \%$ & $-0.4 \%$ & $2.7 \%$ & $-10.3 \%$ & $-1.6 \%$ & & \\
\hline \multicolumn{13}{|l|}{ WPA } \\
\hline Indiana & 4,326 & 4,606 & 4,655 & 4,706 & 4,552 & 4,437 & 4,391 & 4,474 & 3,818 & 3,787 & $-12.5 \%$ & $-1.5 \%$ \\
\hline Iowa & 3,725 & 3,618 & 3,809 & 3,720 & 3,546 & 3,466 & 3,660 & 3,476 & 3,023 & 3,135 & $-15.8 \%$ & $-1.9 \%$ \\
\hline Kansas & 5,782 & 5,851 & 6,150 & 6,363 & 5,766 & 6,414 & 5,636 & 5,970 & 5,130 & 5,046 & $-12.7 \%$ & $-1.5 \%$ \\
\hline Michigan & 4,306 & 5,209 & 4,517 & 4,496 & 4,367 & 3,807 & 3,643 & 3,415 & 3,117 & 3,076 & $-28.6 \%$ & $-3.7 \%$ \\
\hline Missouri & 5,700 & 5,486 & 5,406 & 5,497 & 4,975 & 5,189 & 4,856 & 4,669 & 4,014 & 3,906 & $-31.5 \%$ & $-4.1 \%$ \\
\hline Nebraska & 4,637 & 4,393 & 3,885 & 4,340 & 4,078 & 4,245 & 3,609 & 2,945 & 3,223 & 2,982 & $-35.7 \%$ & $-4.8 \%$ \\
\hline WPS Total & 4,684 & 4,984 & 4,762 & 4,819 & 4,549 & 4,434 & 4,217 & 4,095 & 3,618 & 3,566 & $-23.9 \%$ & $-3.0 \%$ \\
\hline PCPY & & $6.4 \%$ & $-4.5 \%$ & $1.2 \%$ & $-5.6 \%$ & $-2.5 \%$ & $-4.9 \%$ & $-2.9 \%$ & $-11.6 \%$ & $-1.4 \%$ & & \\
\hline US TOTAL & 3,772 & 3,822 & 3,865 & 3,766 & 3,563 & 3,433 & 3,373 & 3,355 & 3,079 & 3,017 & $-20.0 \%$ & $-2.5 \%$ \\
\hline PCPY & & $1.3 \%$ & $1.1 \%$ & $-2.6 \%$ & $-5.4 \%$ & $-3.7 \%$ & $-1.7 \%$ & $-0.5 \%$ & $-8.2 \%$ & $-2.0 \%$ & & \\
\hline
\end{tabular}

Rate: per 100,000 Medicare beneficiaries; Change: of change from 2009 to 2018; GM - geometric average; PCPY - percentage of change from previous year 
Declining Expenditures for Epidural Procedures in the Medicare Population

Appendix Table 3. Utilizations of lumbar interlaminar and caudal epidural rates in the Medicare population from 2009 to 2018 (2016 Medicare carrier).

\begin{tabular}{|c|c|c|c|c|c|c|c|c|c|c|c|c|}
\hline State name & F2009 & F2010 & F2011 & F2012 & F2013 & F2014 & F2015 & F2016 & F2017 & F2018 & Change & Rate \\
\hline \multicolumn{13}{|l|}{ Cahaba } \\
\hline Alabama & 3,330 & 3,395 & 3,527 & 3,468 & 3,393 & 2,989 & 2,894 & 3,060 & 2,408 & 2,374 & $-28.7 \%$ & $-3.7 \%$ \\
\hline Georgia & 2,290 & 2,132 & 2,168 & 2,050 & 1,686 & 1,527 & 1,403 & 1,318 & 1,207 & 1,107 & $-51.6 \%$ & $-7.7 \%$ \\
\hline Tennessee & 2,362 & 2,155 & 2,001 & 1,899 & 1,568 & 1,540 & 1,504 & 1,397 & 1,342 & 1,219 & $-48.4 \%$ & $-7.1 \%$ \\
\hline Total & 2,596 & 2,480 & 2,475 & 2,377 & 2,097 & 1,915 & 1,826 & 1,797 & 1,560 & 1,467 & $-43.5 \%$ & $-6.1 \%$ \\
\hline PCPY & & $-4.5 \%$ & $-0.2 \%$ & $-4.0 \%$ & $-11.8 \%$ & $-8.7 \%$ & $-4.7 \%$ & $-1.6 \%$ & $-13.2 \%$ & $-5.9 \%$ & & \\
\hline \multicolumn{13}{|l|}{ CGS } \\
\hline Kentucky & 3,134 & 3,705 & 3,489 & 3,489 & 3,243 & 2,964 & 2,709 & 2,719 & 2,431 & 2,334 & $-25.5 \%$ & $-3.2 \%$ \\
\hline Ohio & 1,999 & 1,825 & 1,765 & 1,838 & 1,706 & 1,547 & 1,366 & 1,444 & 1,220 & 1,150 & $-42.5 \%$ & $-5.9 \%$ \\
\hline Total & 2,322 & 2,362 & 2,259 & 2,312 & 2,149 & 1,955 & 1,752 & 1,808 & 1,565 & 1,487 & $-36.0 \%$ & $-4.8 \%$ \\
\hline PCPY & & $1.7 \%$ & $-4.4 \%$ & $2.4 \%$ & $-7.1 \%$ & $-9.0 \%$ & $-10.4 \%$ & $3.2 \%$ & $-13.5 \%$ & $-5.0 \%$ & & \\
\hline \multicolumn{13}{|l|}{ First Coast } \\
\hline Florida & 2,302 & 2,217 & 2,038 & 1,971 & 1,887 & 1,682 & 1,545 & 1,599 & 1,385 & 1,325 & $-42.4 \%$ & $-5.9 \%$ \\
\hline PCPY & & $-3.7 \%$ & $-8.1 \%$ & $-3.3 \%$ & $-4.2 \%$ & $-10.9 \%$ & $-8.2 \%$ & $3.5 \%$ & $-13.4 \%$ & $-4.3 \%$ & & \\
\hline \multicolumn{13}{|l|}{ NGS } \\
\hline Connecticut & 1,573 & 1,663 & 1,914 & 1,582 & 1,529 & 1,359 & 1,472 & 1,447 & 1,155 & 1,045 & $-33.6 \%$ & $-4.4 \%$ \\
\hline Illinois & 2,073 & 2,089 & 2,241 & 2,200 & 2,240 & 1,841 & 1,735 & 1,726 & 1,553 & 1,412 & $-31.9 \%$ & $-4.2 \%$ \\
\hline Maine & 2,300 & 2,099 & 2,246 & 2,127 & 2,207 & 2,263 & 1,839 & 1,671 & 1,447 & 1,081 & $-53.0 \%$ & $-8.0 \%$ \\
\hline Massachusetts & 1,705 & 1,657 & 1,807 & 1,778 & 1,952 & 1,774 & 1,778 & 1,782 & 1,682 & 1,583 & $-7.1 \%$ & $-0.8 \%$ \\
\hline Minnesota & 1,155 & 1,066 & 1,030 & 1,034 & 1,074 & 856 & 825 & 704 & 571 & 466 & $-59.7 \%$ & $-9.6 \%$ \\
\hline New Hampshire & 2,300 & 2,401 & 2,629 & 2,152 & 2,369 & 1,994 & 1,894 & 2,074 & 1,594 & 1,792 & $-22.1 \%$ & $-2.7 \%$ \\
\hline New York & 1,139 & 1,099 & 1,143 & 1,127 & 1,121 & 1,006 & 1,054 & 1,018 & 891 & 920 & $-19.3 \%$ & $-2.3 \%$ \\
\hline Rhode Island & 1,487 & 1,629 & 1,444 & 1,369 & 1,111 & 1,398 & 1,411 & 1,190 & 841 & 1,193 & $-19.8 \%$ & $-2.4 \%$ \\
\hline Vermont & 1,427 & 1,417 & 1,274 & 1,278 & 1,651 & 1,439 & 1,623 & 1,294 & 1,178 & 1,184 & $-17.0 \%$ & $-2.0 \%$ \\
\hline Wisconsin & 1,913 & 1,803 & 1,689 & 1,636 & 1,528 & 1,319 & 1,137 & 1,293 & 1,144 & 1,115 & $-41.7 \%$ & $-5.8 \%$ \\
\hline Total & 1,580 & 1,551 & 1,620 & 1,560 & 1,584 & 1,386 & 1,353 & 1,336 & 1,173 & 1,123 & $-28.9 \%$ & $-3.7 \%$ \\
\hline PCPY & & $-1.9 \%$ & $4.5 \%$ & $-3.7 \%$ & $1.5 \%$ & $-12.5 \%$ & $-2.4 \%$ & $-1.3 \%$ & $-12.2 \%$ & $-4.2 \%$ & & \\
\hline \multicolumn{13}{|l|}{ Noridain } \\
\hline Alaska & 1,595 & 1,614 & 1,481 & 1,818 & 1,242 & 1,871 & 1,993 & 1,502 & 1,154 & 1,663 & $4.3 \%$ & $0.5 \%$ \\
\hline Arizona & 1,721 & 1,881 & 1,702 & 1,913 & 1,957 & 1,469 & 1,437 & 1,331 & 1,189 & 1,170 & $-32.0 \%$ & $-4.2 \%$ \\
\hline California & 1,134 & 1,110 & 1,120 & 1,188 & 1,184 & 1,059 & 1,066 & 994 & 839 & 828 & $-27.0 \%$ & $-3.4 \%$ \\
\hline Idaho & 1,928 & 1,462 & 1,616 & 1,729 & 1,683 & 1,630 & 1,313 & 1,284 & 1,247 & 1,124 & $-41.7 \%$ & $-5.8 \%$ \\
\hline Montana & 2,077 & 2,006 & 2,027 & 1,856 & 1,724 & 1,502 & 1,668 & 1,788 & 1,392 & 1,497 & $-27.9 \%$ & $-3.6 \%$ \\
\hline Nevada & 1,475 & 1,211 & 1,560 & 1,564 & 1,621 & 1,225 & 1,202 & 1,095 & 930 & 875 & $-40.7 \%$ & $-5.6 \%$ \\
\hline North Dakota & 2,611 & 3,202 & 3,307 & 3,086 & 3,433 & 3,554 & 2,758 & 2,860 & 2,498 & 2,115 & $-19.0 \%$ & $-2.3 \%$ \\
\hline Oregon & 687 & 670 & 662 & 691 & 539 & 447 & 480 & 406 & 369 & 385 & $-44.0 \%$ & $-6.2 \%$ \\
\hline South Dakota & 2,082 & 2,006 & 2,338 & 1,971 & 1,894 & 1,812 & 2,248 & 2,088 & 1,644 & 1,638 & $-21.3 \%$ & $-2.6 \%$ \\
\hline Utah & 2,176 & 2,092 & 2,366 & 2,151 & 2,536 & 2,052 & 2,143 & 1,830 & 1,710 & 1,729 & $-20.6 \%$ & $-2.5 \%$ \\
\hline Washington & 1,398 & 1,415 & 1,270 & 1,298 & 1,124 & 1,030 & 929 & 889 & 719 & 766 & $-45.2 \%$ & $-6.5 \%$ \\
\hline Wyoming & 2,071 & 2,173 & 1,782 & 2,165 & 1,750 & 1,605 & 1,491 & 2,125 & 1,511 & 1,696 & $-18.1 \%$ & $-2.2 \%$ \\
\hline Total & 1,327 & 1,312 & 1,310 & 1,365 & 1,346 & 1,172 & 1,157 & 1,083 & 924 & 921 & $-30.6 \%$ & $-4.0 \%$ \\
\hline PCPY & & $-1.1 \%$ & $-0.1 \%$ & $4.2 \%$ & $-1.4 \%$ & $-12.9 \%$ & $-1.3 \%$ & $-6.4 \%$ & $-14.7 \%$ & $-0.3 \%$ & & \\
\hline
\end{tabular}


Pain Physician: January/February 2021 24:1-15

Appendix Table 3. Utilizations of lumbar interlaminar and caudal epidural rates in the Medicare population from 2009 to 2018 (2016 Medicare carrier). (continued)

\begin{tabular}{|c|c|c|c|c|c|c|c|c|c|c|c|c|}
\hline State name & F2009 & F2010 & F2011 & F2012 & F2013 & F2014 & F2015 & F2016 & F2017 & F2018 & Change & Rate \\
\hline \multicolumn{13}{|l|}{ Novitas } \\
\hline Arkansas & 2,306 & 2,525 & 2,606 & 2,781 & 2,652 & 2,451 & 2,538 & 2,688 & 2,493 & 2,715 & $17.7 \%$ & $1.8 \%$ \\
\hline Colorado & 2,007 & 2,071 & 2,072 & 1,840 & 1,637 & 1,623 & 1,373 & 1,480 & 991 & 1,012 & $-49.6 \%$ & $-7.3 \%$ \\
\hline Delaware & 3,416 & 3,763 & 4,187 & 3,692 & 4,801 & 3,768 & 3,958 & 3,642 & 3,405 & 3,803 & $11.3 \%$ & $1.2 \%$ \\
\hline $\begin{array}{l}\text { District of } \\
\text { Columbia }\end{array}$ & 400 & 402 & 390 & 725 & 860 & 615 & 638 & 831 & 527 & 438 & $9.4 \%$ & $1.0 \%$ \\
\hline Louisiana & 1,820 & 1,672 & 1,833 & 1,643 & 1,754 & 1,570 & 1,773 & 1,644 & 1,243 & 1,201 & $-34.0 \%$ & $-4.5 \%$ \\
\hline Maryland & 1,696 & 1,600 & 1,841 & 1,885 & 1,621 & 1,571 & 1,762 & 1,656 & 1,378 & 1,562 & $-7.9 \%$ & $-0.9 \%$ \\
\hline Mississippi & 2,808 & 2,828 & 2,977 & 3,038 & 2,765 & 2,818 & 2,853 & 2,641 & 2,148 & 2,403 & $-14.4 \%$ & $-1.7 \%$ \\
\hline New Jersey & 1,555 & 1,322 & 1,429 & 1,442 & 1,335 & 1,324 & 1,159 & 1,146 & 1,007 & 989 & $-36.4 \%$ & $-4.9 \%$ \\
\hline New Mexico & 1,507 & 1,180 & 1,141 & 1,345 & 1,146 & 1,014 & 1,123 & 1,309 & 947 & 878 & $-41.7 \%$ & $-5.8 \%$ \\
\hline Oklahoma & 2,376 & 2,506 & 2,798 & 2,805 & 2,637 & 2,480 & 2,522 & 2,405 & 2,090 & 2,261 & $-4.8 \%$ & $-0.5 \%$ \\
\hline Pennsylvania & 1,663 & 1,745 & 1,777 & 1,738 & 1,742 & 1,523 & 1,577 & 1,473 & 1,382 & 1,391 & $-16.3 \%$ & $-2.0 \%$ \\
\hline Texas & 1,788 & 1,638 & 1,746 & 1,594 & 1,491 & 1,283 & 1,326 & 1,278 & 1,060 & 1,019 & $-43.0 \%$ & $-6.0 \%$ \\
\hline Total & 1,830 & 1,777 & 1,882 & 1,830 & 1,740 & 1,587 & 1,613 & 1,568 & 1,321 & 1,349 & $-26.3 \%$ & $-3.3 \%$ \\
\hline PCPY & & $-2.9 \%$ & $5.9 \%$ & $-2.8 \%$ & $-4.9 \%$ & $-8.8 \%$ & $1.6 \%$ & $-2.8 \%$ & $-15.7 \%$ & $2.1 \%$ & & \\
\hline \multicolumn{13}{|l|}{ Palmetto GBA } \\
\hline North Carolina & 2,338 & 2,267 & 2,375 & 2,553 & 2,234 & 1,809 & 1,714 & 1,794 & 1,511 & 1,417 & $-39.4 \%$ & $-5.4 \%$ \\
\hline South Carolina & 3,305 & 3,415 & 3,320 & 3,345 & 3,218 & 2,658 & 2,878 & 2,754 & 2,444 & 2,445 & $-26.0 \%$ & $-3.3 \%$ \\
\hline Virginia & 1,995 & 1,850 & 1,861 & 1,652 & 1,684 & 1,502 & 1,466 & 1,463 & 1,373 & 1,310 & $-34.3 \%$ & $-4.6 \%$ \\
\hline West Virginia & 1,177 & 1,116 & 1,212 & 1,189 & 1,225 & 856 & 960 & 988 & 776 & 760 & $-35.4 \%$ & $-4.7 \%$ \\
\hline Total & 2,312 & 2,260 & 2,299 & 2,310 & 2,181 & 1,807 & 1,817 & 1,821 & 1,601 & 1,546 & $-33.2 \%$ & $-4.4 \%$ \\
\hline PCPY & & $-2.3 \%$ & $1.7 \%$ & $0.5 \%$ & $-5.6 \%$ & $-17.2 \%$ & $0.6 \%$ & $0.2 \%$ & $-12.1 \%$ & $-3.5 \%$ & & \\
\hline \multicolumn{13}{|l|}{ WPS } \\
\hline Indiana & 2,509 & 2,645 & 2,579 & 2,564 & 2,453 & 2,314 & 2,090 & 2,095 & 1,831 & 1,882 & $-25.0 \%$ & $-3.1 \%$ \\
\hline Iowa & 2,647 & 2,636 & 2,792 & 2,635 & 2,504 & 2,355 & 2,424 & 2,185 & 1,799 & 1,959 & $-26.0 \%$ & $-3.3 \%$ \\
\hline Kansas & 3,333 & 3,277 & 3,642 & 3,588 & 3,414 & 3,528 & 3,027 & 3,199 & 2,922 & 2,837 & $-14.9 \%$ & $-1.8 \%$ \\
\hline Michigan & 2,262 & 2,600 & 2,283 & 2,363 & 2,170 & 1,648 & 1,648 & 1,536 & 1,401 & 1,401 & $-38.1 \%$ & $-5.2 \%$ \\
\hline Missouri & 3,325 & 3,152 & 3,119 & 3,083 & 2,928 & 2,701 & 2,542 & 2,411 & 2,022 & 1,937 & $-41.7 \%$ & $-5.8 \%$ \\
\hline Nebraska & 2,736 & 2,738 & 2,392 & 2,420 & 2,288 & 2,258 & 1,745 & 1,218 & 1,269 & 1,384 & $-49.4 \%$ & $-7.3 \%$ \\
\hline Total & 2,694 & 2,794 & 2,695 & 2,691 & 2,532 & 2,270 & 2,127 & 2,025 & 1,784 & 1,793 & $-33.5 \%$ & $-4.4 \%$ \\
\hline PCPY & & & $-3.6 \%$ & $-0.1 \%$ & $-5.9 \%$ & $-10.4 \%$ & $-6.3 \%$ & $-4.8 \%$ & $-11.9 \%$ & $0.5 \%$ & & \\
\hline US Total & 1,914 & 1,888 & 1,889 & 1,831 & 1,728 & 1,531 & 1,489 & 1,468 & 1,300 & 1,277 & $-33.3 \%$ & $-4.4 \%$ \\
\hline PCPY & & $-1.4 \%$ & $0.1 \%$ & $-3.1 \%$ & $-5.6 \%$ & $-11.4 \%$ & $-2.8 \%$ & $-1.4 \%$ & $-11.5 \%$ & $-1.8 \%$ & & \\
\hline
\end{tabular}

Rate: per 100,000 Medicare beneficiaries; Change: of change from 2009 to 2018; GM - geometric average; PCPY - percentage of change from previous year 
Declining Expenditures for Epidural Procedures in the Medicare Population

Appendix Table 4. Utilizations of lumbar transforaminal epidurals rates in the Medicare population from 2009 to 2018 (2016 Medicare carrier).

\begin{tabular}{|c|c|c|c|c|c|c|c|c|c|c|c|c|}
\hline State name & F2009 & F2010 & F2011 & F2012 & F2013 & F2014 & F2015 & F2016 & F2017 & F2018 & Change & Rate \\
\hline \multicolumn{13}{|l|}{ Cahaba } \\
\hline Alabama & 1,506 & 1,292 & 1,606 & 1,556 & 1,507 & 1,763 & 1,664 & 1,798 & 1,545 & 1,508 & $0.2 \%$ & $0.0 \%$ \\
\hline Georgia & 2,211 & 2,170 & 2,251 & 2,290 & 1,999 & 2,006 & 1,991 & 1,924 & 1,910 & 1,822 & $-17.6 \%$ & $-2.1 \%$ \\
\hline Tennessee & 1,100 & 1,036 & 1,132 & 1,085 & 947 & 990 & 978 & 1,124 & 941 & 986 & $-10.4 \%$ & $-1.2 \%$ \\
\hline Total & 1,644 & 1,551 & 1,702 & 1,691 & 1,515 & 1,601 & 1,567 & 1,626 & 1,497 & 1,467 & $-10.8 \%$ & $-1.3 \%$ \\
\hline PCPY & & $-5.7 \%$ & $9.7 \%$ & $-0.7 \%$ & $-10.4 \%$ & $5.7 \%$ & $-2.2 \%$ & $3.8 \%$ & $-7.9 \%$ & $-2.0 \%$ & & \\
\hline \multicolumn{13}{|l|}{ CGS } \\
\hline Kentucky & 517 & 703 & 718 & 736 & 652 & 664 & 725 & 728 & 846 & 947 & $83.3 \%$ & $7.0 \%$ \\
\hline Ohio & 1,277 & 1,300 & 1,265 & 1,231 & 1,151 & 1,189 & 1,198 & 1,324 & 1,130 & 1,046 & $-18.1 \%$ & $-2.2 \%$ \\
\hline Total & 1,061 & 1,129 & 1,108 & 1,089 & 1,007 & 1,038 & 1,062 & 1,153 & 1,049 & 1,017 & $-4.1 \%$ & $-0.5 \%$ \\
\hline PCPY & & $6.5 \%$ & $-1.8 \%$ & $-1.8 \%$ & $-7.5 \%$ & $3.0 \%$ & $2.3 \%$ & $8.6 \%$ & $-9.0 \%$ & $-3.0 \%$ & & \\
\hline \multicolumn{13}{|l|}{ First Coast } \\
\hline Florida & 1,625 & 1,634 & 1,555 & 1,549 & 1,346 & 1,575 & 1,522 & 1,527 & 1,355 & 1,360 & $-16.3 \%$ & $-2.0 \%$ \\
\hline PCPY & & $0.5 \%$ & $-4.8 \%$ & $-0.4 \%$ & $-13.1 \%$ & $17.0 \%$ & $-3.3 \%$ & $0.3 \%$ & $-11.2 \%$ & $0.4 \%$ & & \\
\hline \multicolumn{13}{|l|}{ NGS } \\
\hline Connecticut & 1,089 & 937 & 1,234 & 1,098 & 1,050 & 1,129 & 915 & 926 & 1,048 & 910 & $-16.4 \%$ & $-2.0 \%$ \\
\hline Illinois & 1,749 & 1,866 & 1,919 & 1,862 & 1,857 & 1,838 & 1,795 & 1,902 & 1,765 & 1,707 & $-2.4 \%$ & $-0.3 \%$ \\
\hline Maine & 1,073 & 815 & 768 & 796 & 502 & 729 & 752 & 685 & 633 & 610 & $-43.2 \%$ & $-6.1 \%$ \\
\hline Massachusetts & 1,062 & 1,133 & 1,210 & 1,362 & 1,210 & 1,191 & 1,179 & 1,110 & 1,018 & 973 & $-8.4 \%$ & $-1.0 \%$ \\
\hline Minnesota & 981 & 934 & 876 & 834 & 879 & 814 & 733 & 673 & 608 & 620 & $-36.8 \%$ & $-5.0 \%$ \\
\hline New Hampshire & 819 & 878 & 1,039 & 1,158 & 872 & 1,327 & 1,271 & 1,142 & 1,367 & 1,096 & $33.8 \%$ & $3.3 \%$ \\
\hline New York & 1,119 & 1,036 & 999 & 1,045 & 1,109 & 1,114 & 1,130 & 1,041 & 954 & 922 & $-17.6 \%$ & $-2.1 \%$ \\
\hline Rhode Island & 843 & 1,137 & 797 & 530 & 429 & 495 & 632 & 472 & 586 & 527 & $-37.5 \%$ & $-5.1 \%$ \\
\hline Vermont & 723 & 789 & 838 & 733 & 934 & 808 & 835 & 837 & 646 & 822 & $13.8 \%$ & $1.4 \%$ \\
\hline Wisconsin & 1,137 & 1,276 & 1,263 & 1,206 & 1,288 & 1,155 & 1,149 & 1,110 & 1,095 & 1,080 & $-5.0 \%$ & $-0.6 \%$ \\
\hline Total & 1,211 & 1,216 & 1,232 & 1,233 & 1,228 & 1,226 & 1,202 & 1,170 & 1,105 & 1,061 & $-12.4 \%$ & $-1.5 \%$ \\
\hline PCPY & & $0.4 \%$ & $1.3 \%$ & $0.1 \%$ & $-0.4 \%$ & $-0.1 \%$ & $-2.0 \%$ & $-2.6 \%$ & $-5.5 \%$ & $-4.0 \%$ & & \\
\hline \multicolumn{13}{|l|}{ Noridain } \\
\hline Alaska & 1,116 & 1,187 & 1,278 & 1,212 & 1,157 & 1,627 & 1,398 & 1,789 & 1,437 & 1,663 & $48.9 \%$ & $4.5 \%$ \\
\hline Arizona & 1,463 & 1,632 & 1,791 & 2,015 & 1,852 & 2,254 & 2,258 & 2,152 & 2,097 & 2,122 & $45.0 \%$ & $4.2 \%$ \\
\hline California & 1,185 & 1,289 & 1,317 & 1,244 & 1,147 & 1,233 & 1,159 & 1,156 & 1,047 & 1,028 & $-13.3 \%$ & $-1.6 \%$ \\
\hline Idaho & 1,054 & 1,201 & 1,692 & 2,067 & 1,394 & 1,570 & 1,594 & 1,737 & 1,325 & 1,355 & $28.6 \%$ & $2.8 \%$ \\
\hline Montana & 1,215 & 1,215 & 1,347 & 1,136 & 1,279 & 1,271 & 1,047 & 1,152 & 1,151 & 1,175 & $-3.3 \%$ & $-0.4 \%$ \\
\hline Nevada & 1,650 & 1,340 & 1,501 & 1,722 & 1,601 & 1,752 & 1,742 & 1,784 & 1,418 & 1,454 & $-11.9 \%$ & $-1.4 \%$ \\
\hline North Dakota & 796 & 750 & 527 & 632 & 395 & 513 & 538 & 555 & 451 & 376 & $-52.8 \%$ & $-8.0 \%$ \\
\hline Oregon & 1,026 & 1,082 & 1,155 & 1,199 & 1,379 & 1,050 & 962 & 864 & 681 & 796 & $-22.5 \%$ & $-2.8 \%$ \\
\hline South Dakota & 1,651 & 1,172 & 1,357 & 1,403 & 1,295 & 1,420 & 1,249 & 1,204 & 1,185 & 1,404 & $-15.0 \%$ & $-1.8 \%$ \\
\hline Utah & 1,884 & 1,760 & 1,676 & 2,117 & 1,942 & 2,294 & 2,492 & 1,969 & 2,070 & 2,127 & $12.9 \%$ & $1.4 \%$ \\
\hline Washington & 1,226 & 1,255 & 1,178 & 1,292 & 1,306 & 1,218 & 1,145 & 1,077 & 1,052 & 909 & $-25.9 \%$ & $-3.3 \%$ \\
\hline Wyoming & 1,151 & 1,698 & 2,075 & 1,927 & 1,843 & 1,895 & 1,793 & 2,272 & 1,923 & 2,016 & $75.2 \%$ & $6.4 \%$ \\
\hline Total & 1,248 & 1,315 & 1,368 & 1,397 & 1,314 & 1,401 & 1,345 & 1,313 & 1,203 & 1,199 & $-4.0 \%$ & $-0.4 \%$ \\
\hline PCPY & & $5.3 \%$ & $4.0 \%$ & $2.1 \%$ & $-6.0 \%$ & $6.6 \%$ & $-4.0 \%$ & $-2.4 \%$ & $-8.3 \%$ & $-0.4 \%$ & & \\
\hline
\end{tabular}


Pain Physician: January/February 2021 24:1-15

Appendix Table 4. Utilizations of lumbar transforaminal epidurals rates in the Medicare population from 2009 to 2018 (2016 Medicare carrier). (continued)

\begin{tabular}{|c|c|c|c|c|c|c|c|c|c|c|c|c|}
\hline State name & F2009 & F2010 & F2011 & F2012 & F2013 & F2014 & F2015 & F2016 & F2017 & F2018 & Change & Rate \\
\hline \multicolumn{13}{|l|}{ Novitas } \\
\hline Arkansas & 846 & 900 & 808 & 688 & 801 & 962 & 918 & 942 & 1,087 & 1,080 & $27.7 \%$ & $2.8 \%$ \\
\hline Colorado & 1,186 & 1,492 & 1,469 & 1,613 & 1,591 & 1,526 & 1,560 & 1,457 & 1,331 & 1,326 & $11.8 \%$ & $1.2 \%$ \\
\hline Delaware & 3,208 & 4,428 & 4,412 & 4,824 & 5,250 & 6,848 & 6,651 & 6,175 & 5,030 & 4,711 & $46.9 \%$ & $4.4 \%$ \\
\hline $\begin{array}{l}\text { District of } \\
\text { Columbia }\end{array}$ & 565 & 978 & 924 & 1,157 & 1,106 & 1,217 & 1,071 & 787 & 589 & 845 & $49.5 \%$ & $4.6 \%$ \\
\hline Louisiana & 2,127 & 1,934 & 2,117 & 1,989 & 1,648 & 1,645 & 1,859 & 1,750 & 1,798 & 1,578 & $-25.8 \%$ & $-3.3 \%$ \\
\hline Maryland & 1,691 & 2,057 & 2,260 & 2,209 & 2,311 & 2,282 & 2,518 & 2,368 & 2,150 & 2,153 & $27.3 \%$ & $2.7 \%$ \\
\hline Mississippi & 1,721 & 2,152 & 2,088 & 1,981 & 1,634 & 1,716 & 1,895 & 1,703 & 1,783 & 1,577 & $-8.4 \%$ & $-1.0 \%$ \\
\hline New Jersey & 1,449 & 1,599 & 1,617 & 1,569 & 1,499 & 1,712 & 1,787 & 1,779 & 1,652 & 1,715 & $18.3 \%$ & $1.9 \%$ \\
\hline New Mexico & 1,251 & 995 & 1,284 & 1,273 & 1,037 & 1,084 & 903 & 987 & 917 & 912 & $-27.0 \%$ & $-3.4 \%$ \\
\hline Oklahoma & 1,690 & 1,813 & 1,939 & 2,003 & 2,066 & 2,295 & 2,301 & 2,182 & 2,274 & 2,195 & $29.9 \%$ & $2.9 \%$ \\
\hline Pennsylvania & 949 & 1,032 & 1,083 & 1,083 & 1,135 & 1,258 & 1,235 & 1,221 & 1,179 & 1,157 & $21.8 \%$ & $2.2 \%$ \\
\hline Texas & 2,055 & 2,188 & 2,281 & 2,024 & 1,865 & 1,913 & 1,900 & 1,823 & 1,698 & 1,452 & $-29.3 \%$ & $-3.8 \%$ \\
\hline Total & 1,545 & 1,688 & 1,761 & 1,680 & 1,608 & 1,704 & 1,735 & 1,676 & 1,596 & 1,501 & $-2.9 \%$ & $-0.3 \%$ \\
\hline PCPY & & $9.2 \%$ & $4.4 \%$ & $-4.6 \%$ & $-4.3 \%$ & $5.9 \%$ & $1.8 \%$ & $-3.4 \%$ & $-4.8 \%$ & $-5.9 \%$ & & \\
\hline \multicolumn{13}{|l|}{ Palmetto GBA } \\
\hline North Carolina & 1,757 & 1,682 & 1,974 & 1,914 & 1,880 & 1,873 & 1,713 & 1,857 & 1,675 & 1,674 & $-4.7 \%$ & $-0.5 \%$ \\
\hline South Carolina & 2,041 & 2,032 & 2,219 & 2,310 & 2,274 & 2,415 & 2,365 & 2,365 & 2,244 & 2,216 & $8.6 \%$ & $0.9 \%$ \\
\hline Virginia & 1,860 & 1,868 & 1,786 & 1,991 & 1,963 & 2,316 & 2,230 & 2,330 & 2,212 & 2,139 & $15.0 \%$ & $1.6 \%$ \\
\hline West Virginia & 938 & 922 & 767 & 995 & 955 & 1,004 & 989 & 945 & 767 & 825 & $-12.1 \%$ & $-1.4 \%$ \\
\hline Total & 1,762 & 1,733 & 1,848 & 1,928 & 1,897 & 2,034 & 1,935 & 2,022 & 1,875 & 1,854 & $5.2 \%$ & $0.6 \%$ \\
\hline PCPY & & $-1.6 \%$ & $6.6 \%$ & $4.4 \%$ & $-1.6 \%$ & $7.2 \%$ & $-4.9 \%$ & $4.5 \%$ & $-7.2 \%$ & $-1.1 \%$ & & \\
\hline \multicolumn{13}{|l|}{ WPS } \\
\hline Indiana & 1,368 & 1,485 & 1,527 & 1,549 & 1,498 & 1,621 & 1,712 & 1,858 & 1,426 & 1,360 & $-0.6 \%$ & $-0.1 \%$ \\
\hline Iowa & 727 & 700 & 723 & 730 & 790 & 734 & 852 & 895 & 863 & 836 & $15.0 \%$ & $1.6 \%$ \\
\hline Kansas & 1,655 & 1,682 & 1,607 & 1,807 & 1,420 & 1,818 & 1,773 & 1,840 & 1,436 & 1,495 & $-9.7 \%$ & $-1.1 \%$ \\
\hline Michigan & 1,404 & 1,865 & 1,583 & 1,495 & 1,566 & 1,604 & 1,423 & 1,393 & 1,296 & 1,237 & $-11.9 \%$ & $-1.4 \%$ \\
\hline Missouri & 1,494 & 1,547 & 1,430 & 1,599 & 1,369 & 1,767 & 1,648 & 1,521 & 1,425 & 1,376 & $-7.9 \%$ & $-0.9 \%$ \\
\hline Nebraska & 1,379 & 1,283 & 1,238 & 1,516 & 1,438 & 1,589 & 1,475 & 1,345 & 1,566 & 1,160 & $-15.9 \%$ & $-1.9 \%$ \\
\hline Total & 1,364 & 1,549 & 1,432 & 1,476 & 1,412 & 1,569 & 1,503 & 1,501 & 1,332 & 1,268 & $-7.0 \%$ & $-0.8 \%$ \\
\hline PCPY & & $13.6 \%$ & $-7.5 \%$ & $3.1 \%$ & $-4.4 \%$ & $11.1 \%$ & $-4.2 \%$ & $-0.2 \%$ & $-11.2 \%$ & $-4.8 \%$ & & \\
\hline US Total & 1,395 & 1,457 & 1,482 & 1,446 & 1,353 & 1,439 & 1,415 & 1,419 & 1,348 & 1,304 & $-6.5 \%$ & $-0.7 \%$ \\
\hline PCPY & & $4.5 \%$ & $1.7 \%$ & $-2.4 \%$ & $-6.4 \%$ & $6.4 \%$ & $-1.7 \%$ & $0.2 \%$ & $-5.0 \%$ & $-3.2 \%$ & & \\
\hline
\end{tabular}

Rate: per 100,000 Medicare beneficiaries; Change: of change from 2009 to 2018; GM - geometric average; PCPY - percentage of change from previous year 